Open Access

\title{
Adaptive modulation and coding in underwater acoustic communications: a machine learning perspective
}

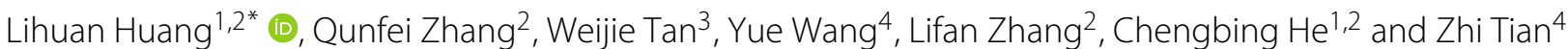

\author{
*Correspondence: \\ hyb504@mail.nwpu.edu.cn \\ ${ }^{1}$ Research and Development \\ Institute, Northwestern \\ Polytechnical University in \\ Shenzhen, Shenzhen, 518057, China \\ 2 School of Marine Science and \\ Technology, Northwestern \\ Polytechnical University, Xi'an, \\ 710072, Shaanxi, China \\ Full list of author information is \\ available at the end of the article
}

\begin{abstract}
The increasing demand for exploring and managing the vast marine resources of the planet has underscored the importance of research on advanced underwater acoustic communication (UAC) technologies. However, owing to the severe characteristics of the oceanic environment, underwater acoustic (UWA) propagation experiences nearly the harshest wireless channels in nature. This article resorts to the perspective of machine learning $(\mathrm{ML})$ to cope with the major challenges of adaptive modulation and coding (AMC) design in UACs. First, we present an ML AMC framework for UACs. Then, we propose an attention-aided $k$-nearest neighbor (A-kNN) algorithm with simplicity and robustness, based on which an ML AMC approach is designed with immunity to channel modeling uncertainty. Leveraging its online learning ability, such A-kNN-based AMC classifier offers salient capabilities of both sustainable self-enhancement and broad applicability to various operation scenarios. Next, aiming at higher implementation efficiency, we take strategies of complexity reduction and present a dimensionality-reduced and data-clustered A-kNN (DRDC-A-kNN) AMC classifier. Finally, we demonstrate that these proposed ML approaches have superior performance over traditional model-based methods by simulations using actual data collected from three lake experiments.
\end{abstract}

Keywords: Underwater acoustic communication (UAC), Harsh oceanic environment, Adaptive modulation and coding (AMC), Machine learning (ML)

\section{Introduction}

Ocean, as the origin of life, covers two thirds of our planet, supports $90 \%$ of the world's freight traffic, and contains a vast amount of underutilized resources. However, human understanding of the deep ocean is even less than space. Therefore, growing attention needs to be cast to researches and exploitations of the mysterious ocean. Recently, thanks to the rapid development of related technologies, underwater acoustic communication (UAC) systems have found broad applications, such as environmental monitoring, offshore exploration, disaster detection, and national security [1].

Traditional UAC systems are generally equipped with a fixed set of physical layer (PHY) parameters, corresponding to a single modulation and coding scheme (MCS). However,

(c) The Author(s). 2020 Open Access This article is licensed under a Creative Commons Attribution 4.0 International License, which permits use, sharing, adaptation, distribution and reproduction in any medium or format, as long as you give appropriate credit to the original author(s) and the source, provide a link to the Creative Commons licence, and indicate if changes were made. The images or other third party material in this article are included in the article's Creative Commons licence, unless indicated otherwise in a credit line to the material. If material is not included in the article's Creative Commons licence and your intended use is not permitted by statutory regulation or exceeds the permitted use, you will need to obtain permission directly from the copyright holder. To view a copy of this licence, visit http://creativecommons.org/licenses/by/4.0/. 
underwater acoustic (UWA) channels are varying temporally and spatially. As a result, it is impossible for an UAC system to cope with a large variety of UWA channel dynamics well by only using one fixed MCS [2-5]. To this end, the adaptive modulation and coding (AMC) technique has emerged to be an appealing avenue for UAC efficiency improvement through tracking channel dynamics and adaptively switching among a set of MCSs to achieve the most efficient transmission.

In 1968, as the origin of the AMC technology, Hayes proposed an adaptive scheme where the transmitter uses the channel state information (CSI) fed back from the receiver to adjust parameters [6]. From then on, lots of research efforts on applying AMC to terrestrial wireless communications have been made. In 1992, Webb presented a variable-rate quadrature amplitude modulation (QAM) system, which offered an attractive solution to the bandwidth restricted microcellular networks [7, 8]. In [9], a bit error rate (BER) comparison was made among various modulation schemes that are used for AMC and then came out with the optimal signal-to-noise ratio (SNR) range of each scheme. In [10], adaptive systems were introduced by evaluating the performance of some simple QAM schemes in both perfectly known and predicted channels. Moreover, in [11], a cross-layer combination of AMC with the truncated automatic repeat request (ARQ) technology was made for the communications of secondary users in cognitive radio networks, which can adapt well to the radio conditions and make full use of the available resources.

Unfortunately, in contrast to terrestrial wireless communications, UACs have to face several unique challenges caused by the undesirable UWA channel characteristics, such as the much more complex spatio-temporal channel variability, more severe multipath fading, and more limited bandwidth [12]. As a result, the development of AMC in UACs is far behind its terrestrial-based counterpart. Some existing results are summarized as follows. Stojanovic used the product of Doppler spread and multipath spread as a criterion for switching between coherent and non-coherent communication modes [13]. For UWA orthogonal frequency-division multiplexing (OFDM) systems, Wan et al. utilized the effective signal-to-noise ratio (ESNR) as a new performance metric for AMC [14]. In [15], Shen et al. selected SNR as the switching metric and presented an adaptive multimode orthogonal multicarrier (MOMC) technology.

So far, the underwater AMC researches have generally focused on the model-based methods. Unfortunately, although extensive efforts have been put on UWA channel modeling, there is not a general channel model yet that fits accurately in various practical scenarios (detailed analysis will be given in Section 2.1), due to the high uncertainty and complexity of UWA channels. As such, those model-based AMC methods can be either insufficient or inaccurate in practical UAC scenarios. To address this problem, we resort to the data-driven machine learning $(\mathrm{ML})$ technology to empower underwater AMC with intelligence, so as to offer immunity to channel modeling uncertainty and thus enabling flexible system optimization and sustainable performance improvement. The ML methods can make predictions or decisions from data observations without the aid of a specific model.

The recent revival of the ML technology has found its wide applications in broad fields, including image/audio processing, economics, and computational biology [16]. Moreover, there are also some interesting results obtained by introducing ML into the field of communications. In terrestrial radios, deep learning (DL) has been advocated for demodulation in OFDM systems [17]. For 5G wireless systems, an efficient online CSI 
prediction scheme which learns the historical data via deep neural networks (DNNs) has been designed [18]. For non-cooperative communication systems, a DL-based method was proposed to perform automatic modulation classification [19], while for UACs, an adaptive and energy-efficient routing protocol for underwater delay/disruption tolerant sensor networks has been proposed [20]. Moreover, NATO has developed a decision treebased approach that is capable of choosing the modulation scheme with the highest data rate among several predefined single-carrier signals depending on CSI [21]. In [22], a reinforcement learning-based adaptive transmission strategy was presented for time-varying UWA channels, which formulates the adaptive problem as a partially observable Markov decision process. These early successes illuminate the feasibility and potential benefits of applying ML in wireless communication systems.

In this paper, we focus on a novel ML-based AMC framework for UACs. Therein, the AMC procedure is formulated as a classifier that has been trained by a pre-organized and labeled database (i.e., training set). After performing model training to establish the functional mapping, we treat such a classifier as a black box, with the input being the real-time channel state and the output being the corresponding optimal MCS. Further, we adopt an online learning mechanism to enable continuous classifier updating during the AMC operation. In doing so, our strategy has salient capabilities of both sustainable selfenhancement and broad applicability to diverse UAC scenarios. The main contribution of this paper can be summarized as follows:

- This paper resorts to the perspective of ML and gives a complete ML AMC framework for UACs, which consists of not only the specific classification algorithm but also the procedure of data preprocessing and labeling. The latter is essential to the success of ML but is often overlooked in generic ML literature.

- A new online learning attention-aided $k$-nearest neighbor (A-kNN) AMC classifier based on supervised learning is proposed, which enables a novel implementation of AMC with immunity to channel modeling uncertainty.

- Aiming at higher implementation efficiency, we further design an improved approach called the dimensionality-reduced and data-clustered A- $k N N$ (DRDC-A- $k N N$ ) AMC classifier, which yields lower complexity by performing feature dimensionality reduction and training set condensation.

- The above contributions have been verified by extensive simulations using actual data collected from lake experiments.

The remainder of this paper is organized as follows. Section 2 analyzes the reason for lacking a general UWA channel model and then defines the system model of ML-based AMC. Section 3 describes our proposed A- $k$ NN-based AMC method. Section 4 focuses on the implementation efficiency improvement of A- $k \mathrm{NN}$-based method and designs the DRDC-A-kNN AMC classifier. Section 5 presents the simulation results. Finally, Section 6 concludes this paper and discusses some future directions.

\section{System model}

In this section, we first explore the reason for the current lack of a general model for UWA channels. Then, we define the system model of AMC in UACs. Next, we formulate the AMC procedure as a classification problem from an ML perspective and discuss the considered ML algorithm, followed by an introduction of the MCSs to be used. 


\subsection{Analysis of UWA channel model}

Since almost all electromagnetic frequencies are severely absorbed and dispersed in water, underwater information transmission is conducted dominantly by acoustic waves [23]. As summarized in Table 1, UWA channels suffer from much more complicated distortions and interferences compared with its terrestrial wireless counterpart and thus posing serious performance-degrading factors to UACs.

Recently, due to its interpretability and simplicity, the ray-tracing model is widely used to formulate the propagation of UWA waves, which assumes that the sound energy propagates along some eigenrays from the source (i.e., transmitter, denoted by TX) to the destination (i.e., receiver, denoted by RX). Therein, following Snell's law, acoustic rays always bend toward the region with lower propagation velocity. Let $T, S$, and $z$ denote temperature, salinity, and depth, respectively, we can calculate the speed of UWA waves (denoted by $c$ ) empirically as

$$
\begin{aligned}
c= & 1448.96+4.591 T-5.304 \times 10^{-2} T^{2}+2.374 \times 10^{-4} T^{3}+1.340(S-35) \ldots \\
& +1.630 \times 10^{-2} z+1.675 \times 10^{-7} z^{2}-1.025 \times 10^{-2} T(S-35) \ldots \\
& -7.139 \times 10^{-13} \mathrm{Tz}^{3} .
\end{aligned}
$$

Such formula reveals that any change in these specific measurements crucial for UAC will result in variation of $c$, which can induce refraction of acoustic ray paths [3, 24]. Consequently, the complexity of UWA propagation comes from the irregularity of the sound speed profile (SSP), which shows the speed of sound in water at different vertical levels. However, as marine environment is a typical inhomogeneous medium with strong dynamic characteristics of seasonal changes and day-night temperature variations, there is still no widely accepted method that can effectively and accurately predict the complicated SSP variations [25]. Such complexity further makes it quite challenging to construct accurate and general UWA channel models in an affordable manner.

\subsection{System model for AMC in UACs}

Considering a node-to-node UAC link from the TX to the RX, we define the system model

\begin{tabular}{|c|c|c|}
\hline Characteristic & Electromagnetic waves in the air & UWA waves \\
\hline Medium dependence & $\begin{array}{l}\text { Propagate regardless of medium, even } \\
\text { in vacuum }\end{array}$ & Must rely on medium vibration \\
\hline Propagation uniformity & $\begin{array}{l}\text { Generally along a straight line, at a stable } \\
\text { speed }\end{array}$ & $\begin{array}{l}\text { Along a curve, with speed greatly } \\
\text { affected by temperature }\end{array}$ \\
\hline Absorption loss under water & 3 dB/m@10kHz & $1.1 \mathrm{~dB} / \mathrm{km} @ 10 \mathrm{kHz}$ \\
\hline Speed in the air & $3 \times 10^{8} \mathrm{~m} / \mathrm{s}$ & $340 \mathrm{~m} / \mathrm{s}$ \\
\hline Speed under water & $2.25 \times 10^{8} \mathrm{~m} / \mathrm{s}$ & $1490 \mathrm{~m} / \mathrm{s}$ \\
\hline $\begin{array}{l}\text { Typical working frequency and } \\
\text { wavelength }\end{array}$ & $\begin{array}{l}\text { GSM_frequency, } 900 \mathrm{MHz} \text {; wavelength, } \\
0.33 \mathrm{~m}\end{array}$ & $\begin{array}{l}\text { Sonar-frequency, } 5 \mathrm{kHz} \text {; } \\
\text { wavelength, } 0.3 \mathrm{~m}\end{array}$ \\
\hline Communication latency & Small & Large \\
\hline Multipath delay & Small multipath delay & $\begin{array}{l}\text { Large delay (> } 10 \mathrm{~ms} \text { ), across } \\
\text { dozens of symbols. }\end{array}$ \\
\hline Doppler & Small scaling factor $\left(\leq 10^{-5}\right)$ & Large scaling factor $\left(10^{-2}\right)$ \\
\hline Variation in time and space & $\begin{array}{l}\text { Related to change of communication } \\
\text { scenarios and variation in short-wave } \\
\text { ionospheric reflection }\end{array}$ & $\begin{array}{l}\text { Related to rapid changes of waves } \\
\text { and periodic changes of seawater }\end{array}$ \\
\hline
\end{tabular}
of $\mathrm{AMC}$ as depicted in Fig. 1. Once receiving a data frame that has been encapsulated into

Table 1 Comparison of electromagnetic waves in the air and UWA waves 


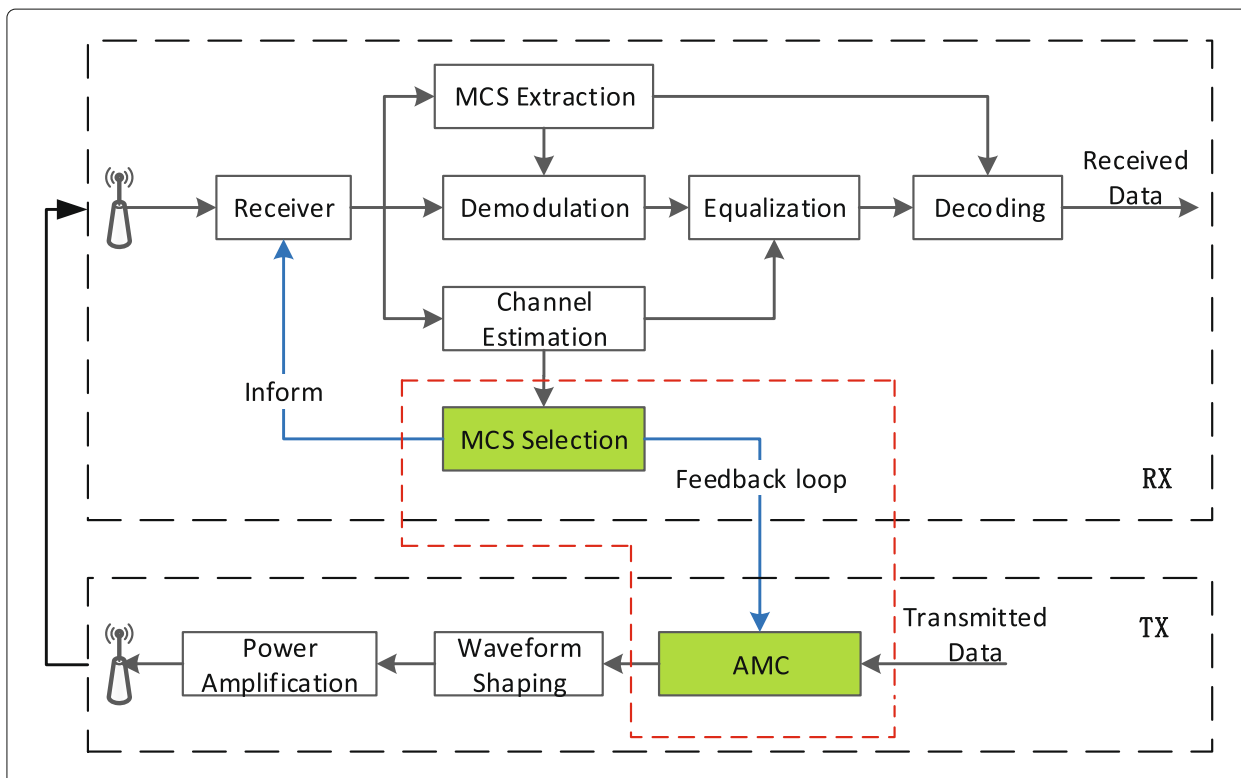

Fig. 1 The system model of AMC in UACS

acoustic waveforms at the $j$ th $(j \in\{1,2, \ldots, J\})$ time instance, RX first performs channel estimation to sense the channel condition $\mathbf{h}_{j} \in \mathcal{H}$, where $\mathcal{H}$ represents the set of all the observed $\mathbf{h}_{j} . \mathbf{h}_{j}$ can be represented by a $P$-dimensional CSI feature set $\mathbf{f}_{j} \in \mathbb{R}^{P}$, in the form of

$$
\mathbf{f}_{j}=\left\{f_{j 1}, f_{j 2}, \ldots, f_{j P}\right\},
$$

where $f_{j p}$ is the $p$ th measured CSI feature. Then, according to the obtained $\mathbf{f}_{j}$, a proper MCS $m_{i} \in \mathcal{M}, i \in\{1,2, \ldots, I\}$ that best matches it will be selected as the optimal solution $m_{\text {opt }}$ under a specific policy $\pi$

$$
m_{\text {opt }}=\left\{m_{i} \in \mathcal{M} \mid \pi\right\},
$$

and then fed back to the TX. Given the harsh UWA channel dynamics, it is necessary to develop and maintain a finite set of allowable MCS realizations (i.e., $\mathcal{M}$ ) for trading off throughput and reliability in practice, where each $m_{i}$ defines a channel coding scheme with rate $R_{c}$ plus a modulation scheme with rate $R_{b}$, and the corresponding actual physical layer data rate $[26,27]$

$$
R_{i}(\text { bps })=R_{b} \times R_{c} .
$$

Next, once notified with a new $m_{\text {opt }}$, the TX will switch to this scheme immediately for subsequent transmissions.

Note that the abovementioned policy $\pi$ is a mapping from channel quality measurements to the MCS to be picked [28]. According to different application scenarios, $\pi$ can aim to either maximize the throughput or minimize the bit error rate (BER). In this paper, for the purpose of maximizing the link throughput $R_{i}$ while satisfying a certain BER constraint $\varphi$ (i.e., $B E R_{i} \leq \varphi$ ), index of the desired solution for a given channel state will be selected depending on

$$
i= \begin{cases}\underset{i}{\arg \max _{i} R_{i},} & \text { if } B E R_{i} \leq \varphi \\ \text { Do not transmit, } & \text { if } B E R_{i}>\varphi .\end{cases}
$$




\subsection{AMC as classification: an ML perspective}

To improve the efficiency of AMC systems, designing an appropriate MCS switching algorithm is of great importance. Existing AMC methods for terrestrial wireless communications can be categorized into two groups: one is based on instantaneous CSI (ICSI) obtained from channel estimation, while the other is based on statistical link information (SLI) inferred through long-term observations or historical knowledge. Unfortunately, due to the complicated SSP variation in the UWA environment, the ICSI-based methods often fail to work effectively for UAC due to the lack of a general channel model that accurately represents complicated UWA propagation effects. Meanwhile, the SLIbased methods hinge on long-term channel statistics and thus suffer severely from slow response speed to fast dynamics and sudden changes in UAC links. These drawbacks of conventional methods motivate us to develop ML-aided AMC approaches for performance improvement.

Turning to the perspective of $\mathrm{ML}$, the AMC procedure can be formulated as a classification problem that aims to partition $\mathbb{R}^{P}$ into nonoverlapping feasible regions for each $m_{i}$. As Fig. 2 depicts, AMC is equivalent to a classifier $G(\cdot)$

$$
G(\cdot): m_{i}=G(\mathbf{f}), \mathbf{f} \in \mathbb{R}^{P} \text {. }
$$

As such, we further propose a novel framework of ML-based AMC for UAC systems. As illustrated in Fig. 3, it is appealing to track and adapt to complex UWA scenarios, with immunity to channel modeling uncertainty.

\subsection{Classification algorithm for ML-based AMC}

Generally, typical ML algorithms can be classified into four broad categories depending on the nature of the dataset for learning or the feedback mechanism available to the learning system. They are supervised learning (SL), unsupervised learning (UL), semi-supervised learning (SSL), and reinforcement learning (RL), where SL algorithms

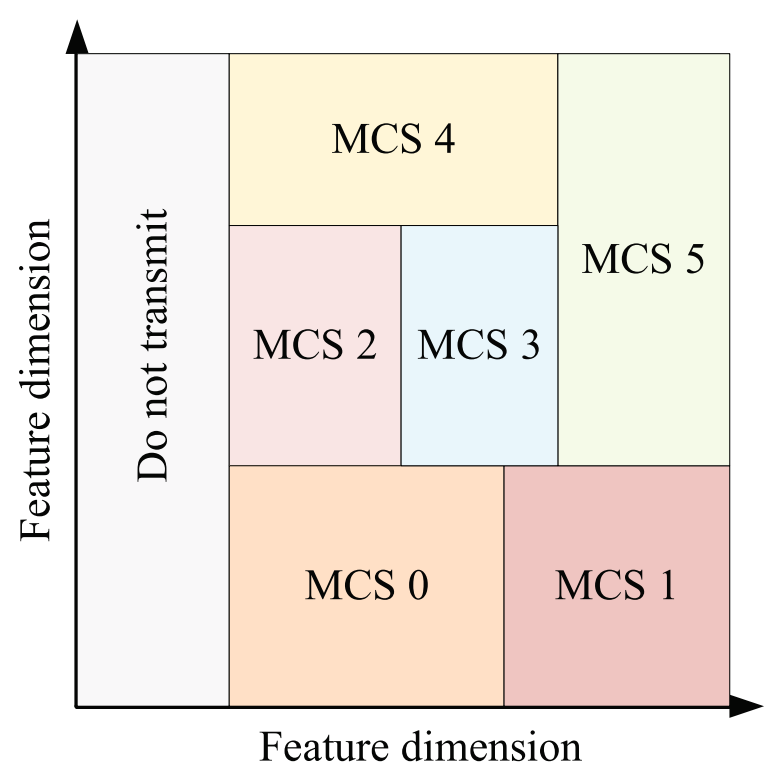

Fig. 2 Partition a two-dimensional feature space into feasible regions for each available MCS 


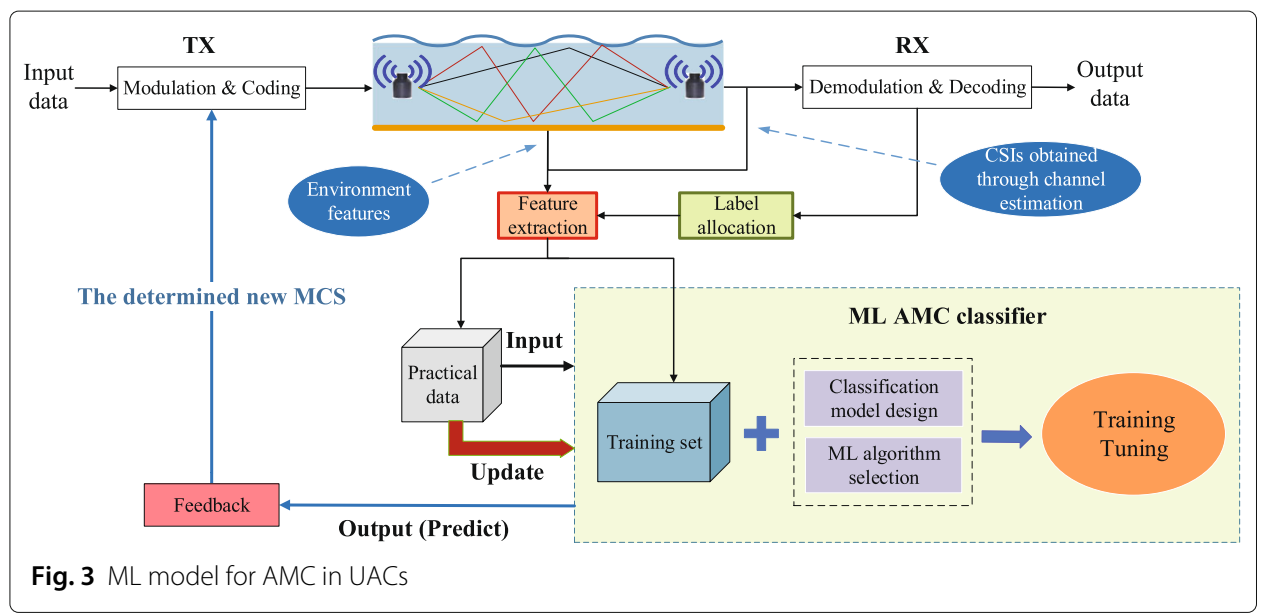

are more convenient at solving classification problems due to their ability to infer an input-output mapping function from labeled training data.

In this paper, we adopt the $k \mathrm{NN}$ algorithm to investigate the potential of ML for AMC in UACs and obtain the AMC classifier $G(\cdot)$. As a non-parametric method among the most popular SL approaches, $k \mathrm{NN}$ is often used as a benchmark for more complex algorithms, such as support vector machine (SVM) and deep neural network (DNN), thanks to its simplicity and robustness that leads to achievable results even facing small training sets [29].

Assume a training set $T$

$$
T=\left\{\left(y_{n}, \mathbf{x}_{n}\right), n=1,2, \ldots, N\right\},
$$

where $y_{n}$ denotes the labeled membership of each observation and $\mathbf{x}_{n}=\left(f_{n 1}, f_{n 2}, \ldots, f_{n P}\right)$ represents the associated feature values. Once given a query $\omega$, the $k \mathrm{NN}$ algorithm first searches in $T$ to find its $k$-nearest neighbors depending on some specific distance measurements $d(\cdot)$, where the Euclidean distance

$$
d\left(\omega, \mathbf{x}_{n}\right)=\left(\sum_{p=1}^{P}\left(f_{n p}-\omega_{p}\right)^{2}\right)^{\frac{1}{2}}
$$

is the one that has been widely utilized. Then, $k \mathrm{NN}$ proceeds to the voting stage and labels $\omega$ with the class $y_{\omega}$ that the majority of the $k$ neighbors belong to. Such a process can be expressed as

$$
y_{\omega}=\arg \max _{y} \sum_{k=1}^{K} \delta\left(y=y_{k}\right),
$$

where $\delta$ is the Dirac function that equals to 1 if $y=y_{k}$ or 0 otherwise.

However, since different distances reflect different degrees of similarity, the information provided by each of the $k$-nearest neighbors to support the classification process is obviously of different importance. Thus, directly adopting the conventional $k \mathrm{NN}$ algorithm where each neighbor has a equal weight in the voting stage will inevitably bring some performance degradation to the classification, or even lead to incorrect results. To address this issue, we resort to the attention mechanism and propose the A- $k \mathrm{NN}$ algorithm for the underwater AMC task. As a cognitive process of selectively concentrating on a few 
features while ignoring others, the attention mechanism can help ML models assign different weights to each part of the input, extract more critical and important information, and make more accurate judgments without incurring more costs to model computation and storage [30, 31].

In the $\mathrm{A}-k \mathrm{NN}$ algorithm, the specific job of the attention mechanism is to produce a set of $w_{k}$ for the concerned neighbors, where $w_{k}$ denotes the weight of the $k$ th-nearest neighbor of $\omega$. Then by assigning nearer neighbors with higher $w_{k}$, attention can dynamically highlight the importance of different neighbors in the voting stage. Thus, we have

$$
y_{\omega}^{A}=\arg \max _{y} \sum_{k=1}^{K} w_{k} \times \delta\left(y=y_{k}\right) .
$$

Note that attention weights can be trained, or predefined based on some sort of correlation metric, or even be Gaussian shaped with tunable parameters. In this work, we set $w_{k}$ to the Squared Inversion (SI) kernel, i.e.,

$$
w_{k}=w\left(d_{k}\right)=\frac{1}{d_{k}^{2}} \text {. }
$$

\subsection{MCS model}

In this work, we adopt the convolutional coded multicarrier multiple frequency shift keying (CC-MC-MFSK) as the transmission scheme to evaluate the proposed ML-based AMC system, where Fig. 4 depicts its structure.

\subsubsection{MC-MFSK}

With the advances in UAC technologies, considerable efforts have been made in the design of modulation schemes. From FSK and phase-shift keying (PSK), through orthogonal frequency-division multiplexing (OFDM), to the latest orthogonal signal-division multiplexing (OSDM) [32-34], these modulation schemes have been investigated extensively and proven useful in the harsh oceanic environment.

In this paper, we adopt the scheme of MC-MFSK, which combines the techniques of MFSK and OFDM to transmit information in parallel over multiple orthogonal subchannels $[35,36]$. As such, this method not only inherits the robust performance of MFSK, but also integrates the high spectral efficiency of OFDM. Moreover, by introducing the

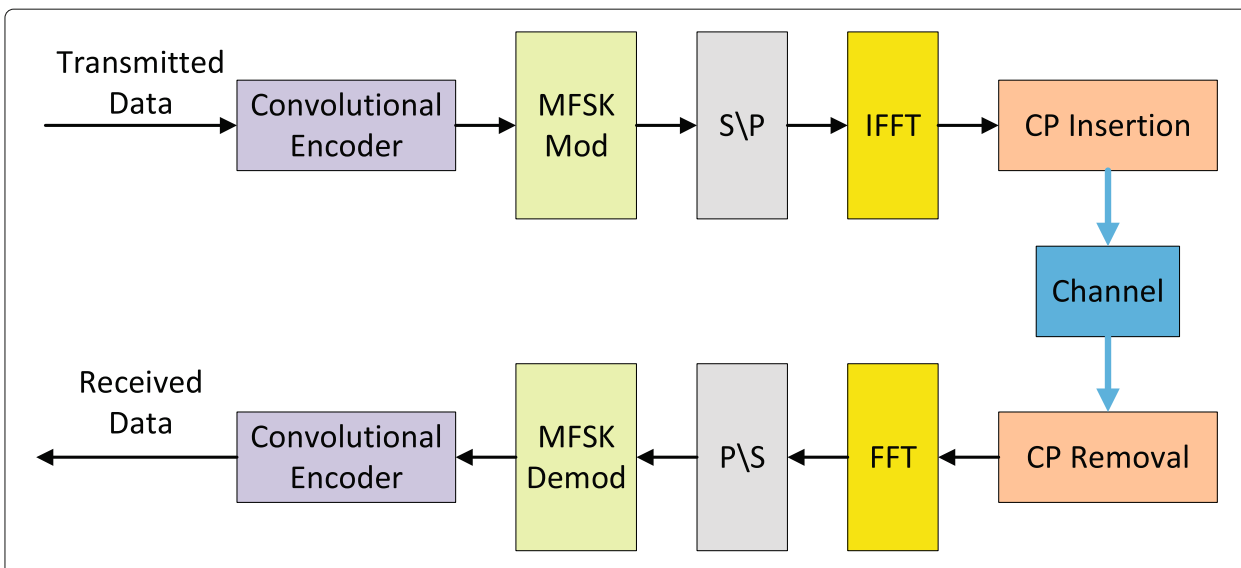

Fig. 4 Block diagram of CC-MC-MFSK 
parameter of frequency diversity, an additional degree of freedom in the MCS table design can be obtained to improve its scope of application.

\subsubsection{Convolutional code}

To reduce the transmission errors caused by the noise and interference in UWA channels, there have been extensive works on the design of error-correcting code (ECC) that can improve the ability of error controlling at the price of adding redundancy to the original message. Among existing ECC approaches, such as Reed-Solomon (RS) codes, low-density parity-check (LDPC) codes, and turbo codes, the simple convolutional code with Viterbi decoder is selected as the coding scheme in the following discussions, thanks to its ability to obtain a good trade-off between error-correcting performance and implementation complexity. To be more specific, the adopted convolutional code is with coding rate $R_{c}=1 / 2$, constraint length 7 , and generator polynomial $(171,133)$.

\section{A-kNN AMC for UAC}

In this section, we present a novel ML framework for AMC in UAC systems, where an online learning A- $k \mathrm{NN}$ classifier serves as the switching method for predicting the optimal MCS to maximize the link throughput.

\subsection{System assumptions}

Specifically, we consider the following assumptions in our A-kNN AMC method:

- Accurate channel knowledge. We assume that through channel estimation, the RX obtains CSI accurately, thus enabling a high-quality training process.

- Perfect feedback. Generally, RX informs TX of the selected MCS by sending a message through the feedback channel. In this paper, we assume an error-free feedback stage.

\subsection{A-kNN classifier}

Figure 5 illustrates the architecture of our A- $k$ NN AMC method, where a two-stage process is conducted. During the offline training stage, the mapping function between the

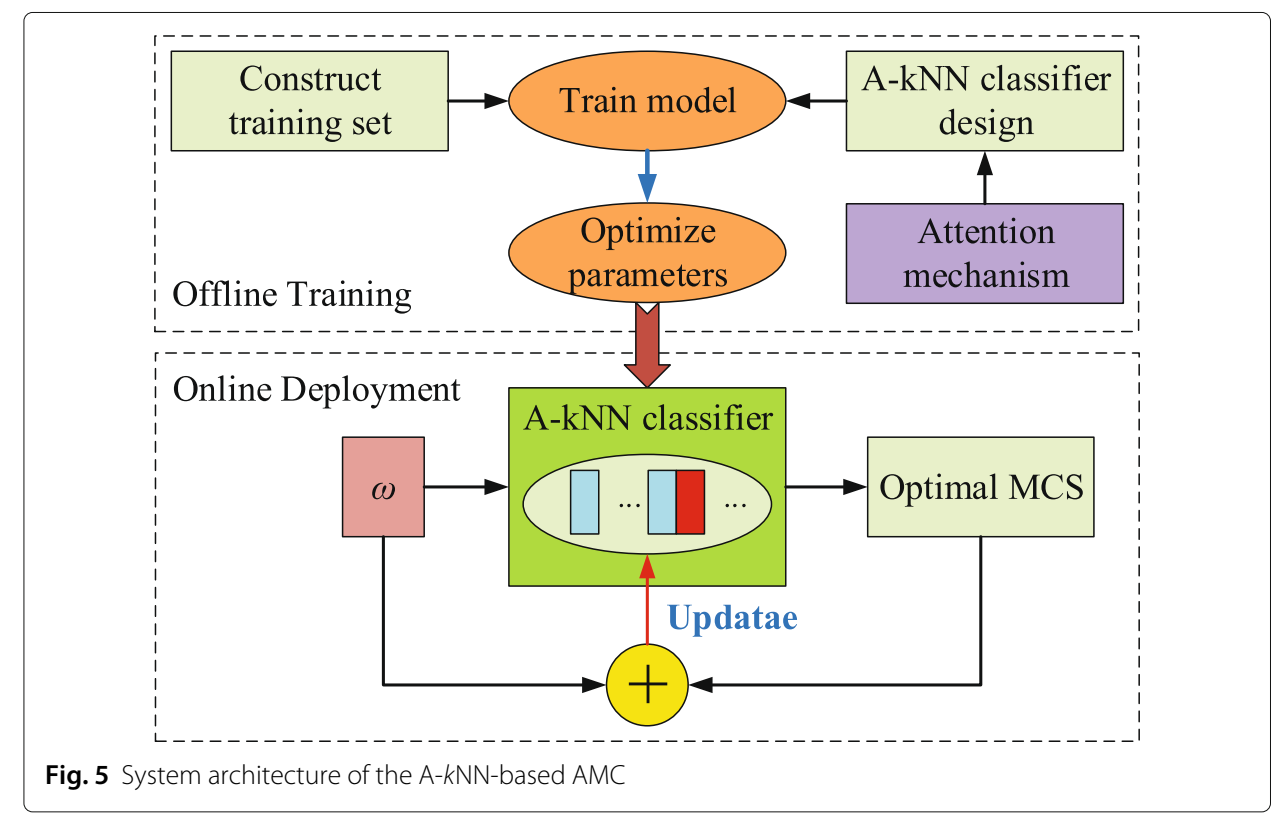


input CSI and the output MCS modes is established by training the A- $k N N$ classifier iteratively until a certain stopping criterion is satisfied, a.k.a. an expected model prediction accuracy. Therein, the training set is constructed based on the signal samples generated from predefined $\mathcal{M}$ for various kinds of $\mathcal{H}$. During the online deployment stage, the trained classifier is applied to analyze the real-time input CSI vector $\omega$ and generate the optimal MCS to best match the practical UAC channel conditions. Further, an online learning mechanism is incorporated to update the AMC classifier as new data arrive, so as to constantly improve the applicability of the model. Then, we summarize the A- $k \mathrm{NN}$ AMC method in Algorithm 1.

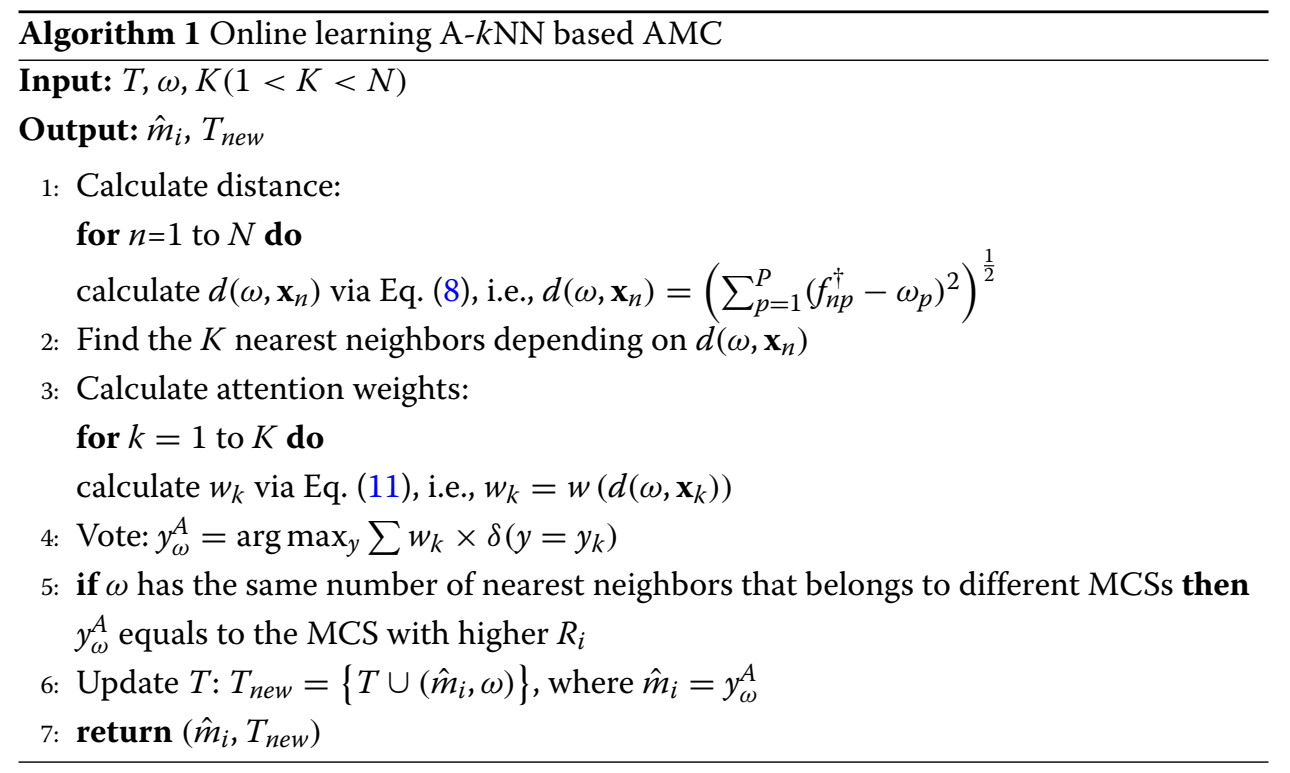

In the framework of the proposed A- $k \mathrm{NN}$ AMC discussed above, there are some critical steps that are needed to be further clarified. Next, we elaborate on two techniques of the model training: feature set selection and training set construction.

\subsection{Feature set selection}

To apply A- $k N N$ to AMC, we start with collecting a set of synthetic and real labeled data from both simulations and field experiments. Without loss of generality, various UWA channel models and test scenarios are used to generate the input channel data, each of which is represented by a $P$-dimensional feature set.

To support a good training accuracy, we assign $P$ with a large value to provide enough information, or the capability of our A- $k$ NN classifier will be restricted seriously. However, due to the so-called curse of dimensionality, each dimensionality added to $\mathbb{R}^{P}$ leads to a significant computational complexity increase in both feature extraction and model training [37]. As such, there is an important trade-off between information sufficiency and computational efficiency. To this end, the current practice is to preset the feature space by experience or prior knowledge. In this work, we construct a six-dimensional feature set $\mathbf{f} \in \mathbb{R}^{6}$ to represent different UWA channel conditions by extracting the following CSI parameters: signal-to-noise ratio (SNR), time delay spread $\left(\tau_{\max }\right)$, time delay of the strongest path $\left(\tau_{\text {hmax }}\right)$, total power of the first three paths $\left(e_{3}\right)$, total power of all paths 
$\left(e_{\text {total }}\right)$, and the normalized amplitude of the first path $\left(\left|h_{1}\right|\right)$. Note that $e_{3}, e_{\text {total }}$, and $\left|h_{1}\right|$ are related to the normalized format of the raw channel impulse response at each observation instance, where the amplitude of each path has been scaled to $[0,1]$ by dividing the absolute value of the strongest path amplitude. Besides, $e_{3}$ and $e_{\text {total }}$ can reflect the energy distribution of the first three paths and of all paths, which is related to the complexity of channel structure.

\subsection{Training set construction}

As the foundation of ML techniques, training data is an essential set of input information that enables ML algorithms to learn the underlying principles and extract key features. For the proposed A- $k N N$ AMC classifier, the constructed training set has to involve the corresponding BER, denoted by $B E R_{i j}\left(m_{i}, \mathbf{f}_{j}\right)$, of each $m_{i}$ in all kinds of $\mathbf{f}_{j}$. Once the required information of each observation

$$
\mathbf{o}_{i j}=\left\{m_{i}, \mathbf{f}_{j}, B E R_{i j}(\cdot) \mid B E R(\cdot) \leq \varphi\right\}
$$

is made available, we first store them in the corresponding subsets according to $m_{i}$, i.e.,

$$
T_{0 m_{i}}=\left\{\left(y_{i}, \mathbf{o}_{i j}\right), j=1,2, \ldots, J\right\},
$$

and then merge all to form the training set:

$$
T_{0}=\left\{\bigcup_{i=1}^{I} T_{0 m_{i}}\right\} .
$$

So far, an original training set has been successfully constructed, as illustrated in Fig. 6 . However, as an important step before the training starts, further preprocessings to $T_{0}$ are needed to turn raw data into a cleaner and more reasonable format for the AMC task.

\subsection{1 "One-to-one" mapping}

Each original $T_{0 m_{i}}$ includes the observation of $m_{i}$ in all the possible channels, thus making the mapping relationship between $\mathcal{M}$ and $\mathcal{H}$, provided by the whole training set, one-tomany. Unfortunately, such mapping relation will significantly confuse the classifier and make it impossible to determine the optimal MCS for each specific $\mathbf{f}_{j}$ through training. To solve this problem, we use Eq. (5) to modify the sets and only retain information of the desired $m_{o p t}$, so as to obtain a one-to-one mapping function for model training. Then, the processed $T_{0 m_{i}}$ can be expressed as

$$
T_{m_{i}}=\left\{\left(y_{i}, \mathbf{o}_{i n_{i}}\right), n_{i}=1,2, \ldots, N_{i}\right\},
$$

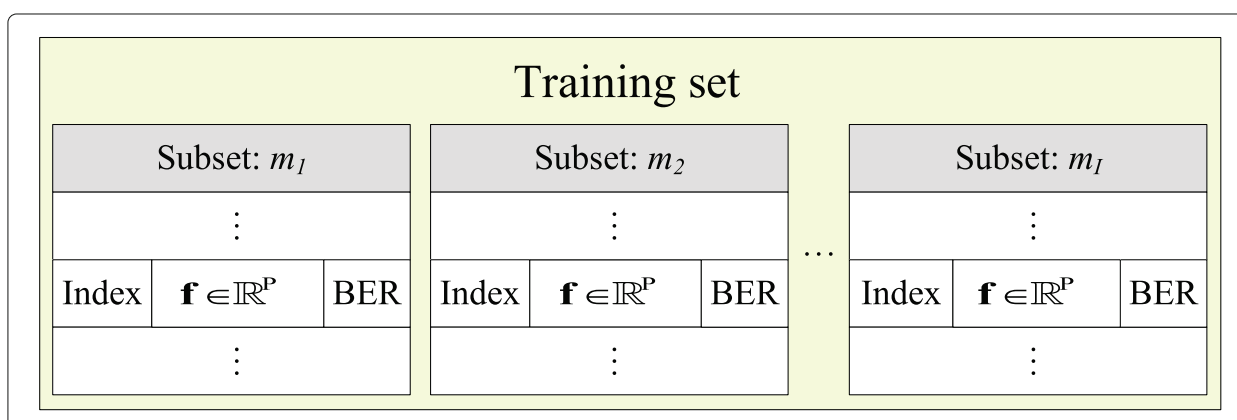

Fig. 6 Structure of the training set 
with $N_{i}$ denoting the number of observations retained in the $i$ th subset.

\subsubsection{Feature scaling}

Since the various features included in $T_{0}$ are almost impossible to have a consistent magnitude, the one with a wider range of value will dominate the distance calculated by A- $k \mathrm{NN}$, which means other features' influences will be overpowered, and thus, significant loss in training accuracy will be caused. To address this issue, we perform feature normalization across all variables. Specifically, for each $f_{p}$, its normalized counterpart $f_{p}^{\dagger}$ can be calculated via

$$
f_{p}^{\dagger}=\frac{f_{p}-f_{p \min }}{f_{\text {pmax }}-f_{\text {pmin }}}
$$

with $f_{\text {pmin }}$ and $f_{\text {pmax }}$ denoting the minimum and maximum values of $f_{p}$, respectively. After feature scaling, a new training set $T$ with normalized feature quantities has been successfully obtained. Let $N=\sum_{i=1}^{I} N_{i}$ represent the total number of observations that belong to different $T_{m_{i}}^{\prime}$, we have the data matrix of the whole channel observations as

$$
\mathbf{x}_{N \times P}=\left[\begin{array}{l}
\mathbf{x}_{1} \\
\mathbf{x}_{2} \\
\vdots \\
\mathbf{x}_{N}
\end{array}\right]=\left[\begin{array}{cccc}
f_{11}^{\dagger} & f_{12}^{\dagger} & \ldots & f_{1 P}^{\dagger} \\
f_{21}^{\dagger} & f_{22}^{\dagger} & \ldots & f_{2 P}^{\dagger} \\
\vdots & \ddots & & \vdots \\
f_{N 1}^{\dagger} & f_{N 2}^{\dagger} & \ldots & f_{N P}^{\dagger}
\end{array}\right] .
$$

Note that all the training sets we discussed in this paper have been preprocessed by the abovementioned two steps.

\section{An efficiency-enhancing A-kNN AMC approach}

With the ability to implicitly learn the uncertain and complex UWA channel, the proposed A- $k \mathrm{NN}$ AMC classifier is demonstrated to achieve higher efficiency and wider applicability than the traditional model-based approaches. However, before deploying it into practical scenarios, we should give enough attention to the inherent complexity of $k \mathrm{NN}$ algorithm and make efforts to improve its implementation efficiency.

\subsection{Complexity of the A-kNN classifier}

The implementation complexity of the A- $k N N$ classifier can be generally divided into the following two major aspects: (1) storage complexity of large amounts of training data and (2) computational complexity in the searching of the nearest neighbors. Specifically, to implement the proposed classifier in practice, a major difficulty is that we have to reserve enough memory to store all the training data. Moreover, since the searching of the nearest neighbors requires computing and sorting the distances from all stored observations, the proposed classifier will be computationally intensive when facing huge amounts of data or high dimensional feature space. All of these adverse characteristics pose significant challenges for the proposed A- $k N N$ AMC method to achieve a good performance in the actual deployment.

\subsection{DRDC-A-kNN classifier}

To overcome the aforementioned challenges, we turn to design an improved approach with lower complexity than previous solution, which is called the DRDC-A- $k$ NN classifier. Figure 7 illustrates the architecture of this new approach by highlighting its 


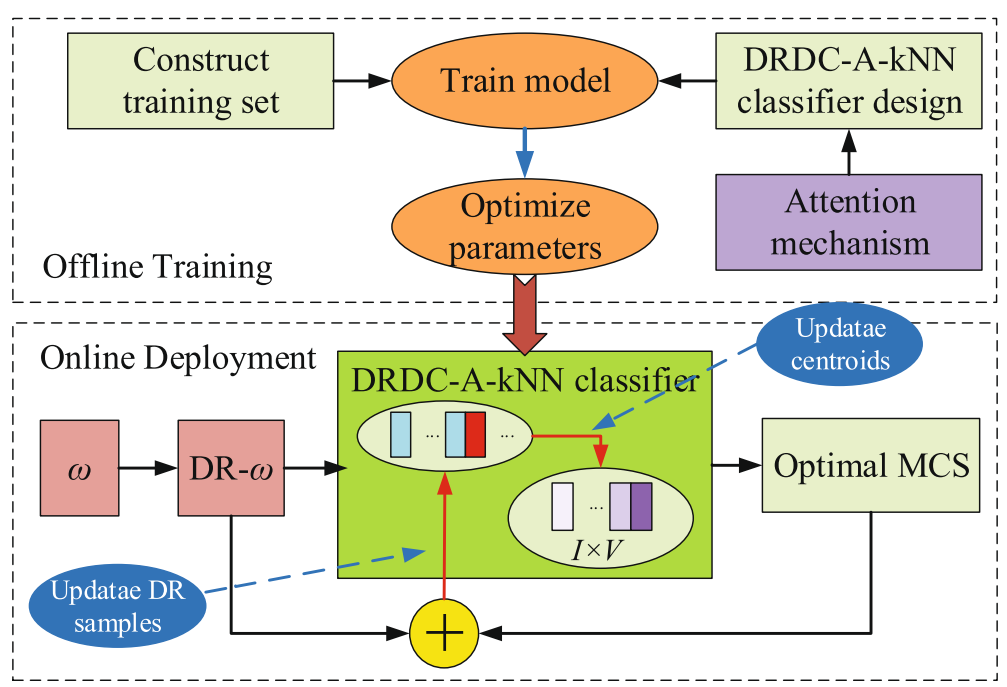

Fig. 7 System architecture of the DRDC-A-kNN AMC

two significant improvements: (1) feature dimensionality reduction through principal component analysis (PCA) and (2) training set condensation via $k$-means data clustering.

\subsubsection{Dimensionality reduction}

As a frequently used technique in data analysis, PCA provides a tool to seek linear combinations of the original variables which retain maximal variance and thus minimize information loss over feature transformation. In the DRDC-A- $k N N$ classifier, we adopt PCA to reduce the complexity induced by high feature dimensionalities. Let $\mathbf{X}^{\prime}$ denote the column-wise centralized form of the original $N \times P$ data matrix $\mathbf{X}$

$$
\mathbf{X}^{\prime}=\left[\begin{array}{l}
\mathbf{x}_{1}^{\prime} \\
\mathbf{x}_{2}^{\prime} \\
\vdots \\
\mathbf{x}_{N}^{\prime}
\end{array}\right]=\left[\begin{array}{cccc}
f_{11}^{\prime} & f_{12}^{\prime} & \ldots & f_{1 P}^{\prime} \\
f_{21}^{\prime} & f_{22}^{\prime} & \ldots & f_{2 P}^{\prime} \\
\vdots & \ddots & & \vdots \\
f_{N 1}^{\prime} & f_{N 2}^{\prime} & \ldots & f_{N P}^{\prime}
\end{array}\right],
$$

which contains $N$ observations with each represented by a $P$-dimensional feature set. We perform singular value decomposition (SVD) to $\mathbf{X}^{\prime}$ and obtain

$$
\mathbf{X}^{\prime}=\mathbf{U} \Sigma \mathbf{V}^{T},
$$

where singular values in $\Sigma_{N \times P}$ are sorted in descending order. Then, the columns of $\mathbf{U}_{N \times N} \Sigma_{N \times P}$ are the principal components (PCs), while PC loadings are represented by the corresponding columns of $\mathbf{V}_{P \times P}$, and sample variance of the $q$ th PC can be calculated as $\Sigma_{q q}^{2} /(N-1)$ [38]. Generally, holding more than an expected ratio $\psi$ of total variance, i.e.,

$$
\frac{\sum_{q=1}^{Q} \Sigma_{q q}^{2}}{\sum_{q=1}^{\min (N, P)} \Sigma_{q q}^{2}} \geq \psi,
$$

the first $Q$ PCs are retained to compactly represent the original data for training. Along this way, a great dimensionality reduction can be achieved by PCA through converting $\mathbf{f} \in \mathbb{R}^{P}$ into a lower dimensional subspace $\overline{\mathbf{f}} \in \mathbb{R}^{Q}$, i.e., 


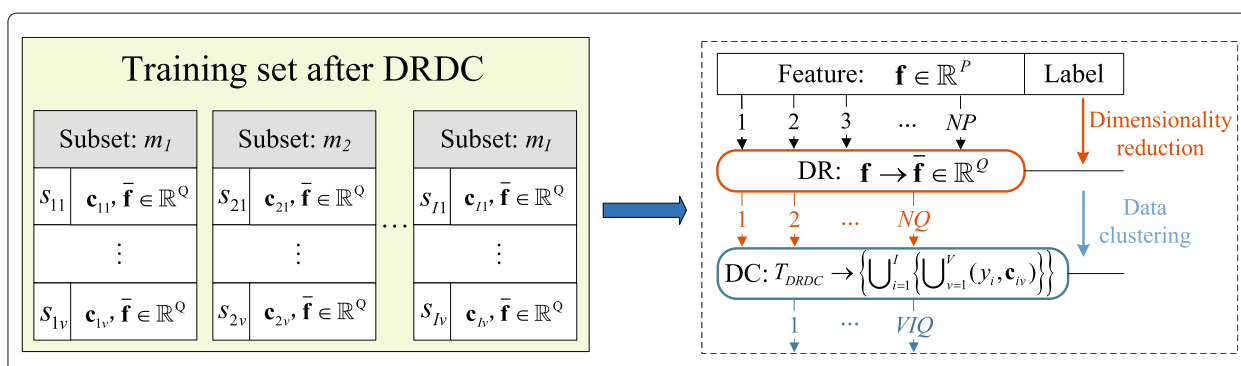

Fig. 8 Training set architecture for the DRDC-A-kNN AMC

$$
\overline{\mathbf{f}}=\mathbf{f} \times \mathbf{V}[:, 1: Q]
$$

\subsubsection{Data clustering}

Another efficiency-improving measure is to cluster each class of training samples and then use only some representative observations for the model training. Considering its efficiency and robustness in cluster analysis, we adopt the $k$-means technique to perform data condensation. Given a training set as depicted in Fig. $6, k$-means is performed in each subset $T_{m_{i}}$ to partition the $N_{i}$ observations into $V\left(V \leq N_{i}\right)$ clusters as

$$
T_{m_{i}}=\left\{\mathbf{s}_{i 1}, \mathbf{s}_{i 2}, \ldots, \mathbf{s}_{i v}\right\}, v=1,2, \ldots, V,
$$

with $\mathbf{c}_{i v}$ denoting the corresponding centroid of each $\mathbf{s}_{i v}$. Specifically, such procedure can be accomplished through proceeding the following two steps iteratively until satisfying the stopping criteria that the assignments no longer change when the centroids are updated:

- Step 1 (data assignment). Assign each observation to the cluster of the nearest $\mathbf{c}_{i v}$, where the squared Euclidean distance is used, i.e.,

$$
s^{(t)}=\underset{\mathbf{c}_{i v} \in \mathbb{C}}{\arg \min _{i n_{i}}}-\mathbf{c}_{i v}^{(t)} \|_{2}^{2},
$$

Note that the initial $\mathbf{c}_{i v}$ are some randomly selected points from $T_{m_{i}}$.

- Step 2 (centroid update). Once an assignment is finished, recalculate the means of the new cluster to update its centroid as

$$
\mathbf{c}_{i v}^{(t+1)}=\frac{1}{\left\|\mathbf{s}^{(t)}\right\|_{0}} \sum_{\mathbf{o}_{i v} \in \mathbf{s}^{(t)}} \mathbf{o}_{i v} .
$$

Table 2 Complexity comparison of A-kNN and DRDC-A-kNN AMC classifiers. $k_{n e w}$ is the selected new $k$ after DRDC, which is equal to the least integer greater than $\sqrt{V I}$

\begin{tabular}{llll}
\hline \multirow{2}{*}{ Comparison } & & \multicolumn{2}{c}{ Algorithm } \\
\cline { 3 - 4 } & & A-kNN & DRDC-A-kNN \\
\hline \multirow{2}{*}{ Processing } & Distance calculation & $O(P N)$ & $O(Q V I)$ \\
& Sorting & $O(N / \log N)$ & $O(V I l o g(V I))$ \\
Memory (bits) & Voting & $O\left(k^{2} \log k\right)$ & $O\left(k_{\text {new }}^{2} /\right.$ ogk $\left.k_{\text {new }}\right)$ \\
\hline
\end{tabular}


Table 3 MCS table

\begin{tabular}{lllllll}
\hline Index & Name & Modulation scheme & Diversity & Coding scheme & $\boldsymbol{R}_{\boldsymbol{c}}$ & $\boldsymbol{R}_{\boldsymbol{i}}(\boldsymbol{b} \boldsymbol{p s})$ \\
\hline 1 & MCS1 & MC-2FSK & 4 & Convolutional code & $1 / 2$ & 227 \\
2 & MCS2 & MC-4FSK & 2 & Uncoded & 1 & 911 \\
3 & MCS3 & MC-8FSK & 1 & Uncoded & 1 & 1366 \\
\hline
\end{tabular}

Finally, using the obtained centroids to represent each corresponding cluster, we successfully obtain an efficient form of the training set

$$
T_{D R R C}=\left\{\bigcup_{i=1}^{I} T_{D R D C-m_{i}}\right\}=\left\{\bigcup_{i=1}^{I}\left\{\bigcup_{v=1}^{V}\left(y_{i}, \mathbf{c}_{i v}\right)\right\}\right\}=\left\{\bigcup_{\bar{n}=1}^{V I}\left(y_{\bar{n}}, \mathbf{c}_{\bar{n}}\right)\right\} .
$$

Figure 8 shows its architecture. Therein, the number of features in the training set is first reduced from $N P$ to $N Q$ via DR, then further decreased to VIQ after DC. Assuming that $b$-bit memory is required for the storage of each feature or label, we compare the complexity of the DRDC-A- $k$ NN AMC classifier and that of the previous A- $k \mathrm{NN}$ approach, as shown in Table 2. Remarkably, this novel design with enhanced computational efficiency is demonstrated to be effective.

\subsubsection{Online learning}

Moreover, withthe ability of online learning, the DRDC-A-kNN AMC method is able to continuously improve its understanding of the UWA environment. Therein, $T_{D R D C}$ is updated through tuning the centroid $\mathbf{c}_{i v}$ of each cluster as the new sample $\omega$ arrives, i.e.,

$$
\mathbf{c}_{i v}^{\left(n_{i v}+1\right)}=\frac{1}{n_{i v}+1}\left(\mathbf{c}_{i v}^{\left(n_{i v}\right)} \times n_{i v}+\bar{\omega}\right),
$$

where $n_{i v}$ is the number of observations included in each cluster before the new arrival, and $\bar{\omega}$ is the DR processed $\omega$. The algorithm of DRDC-A- $k$ NN AMC is summarized in Algorithm 2.
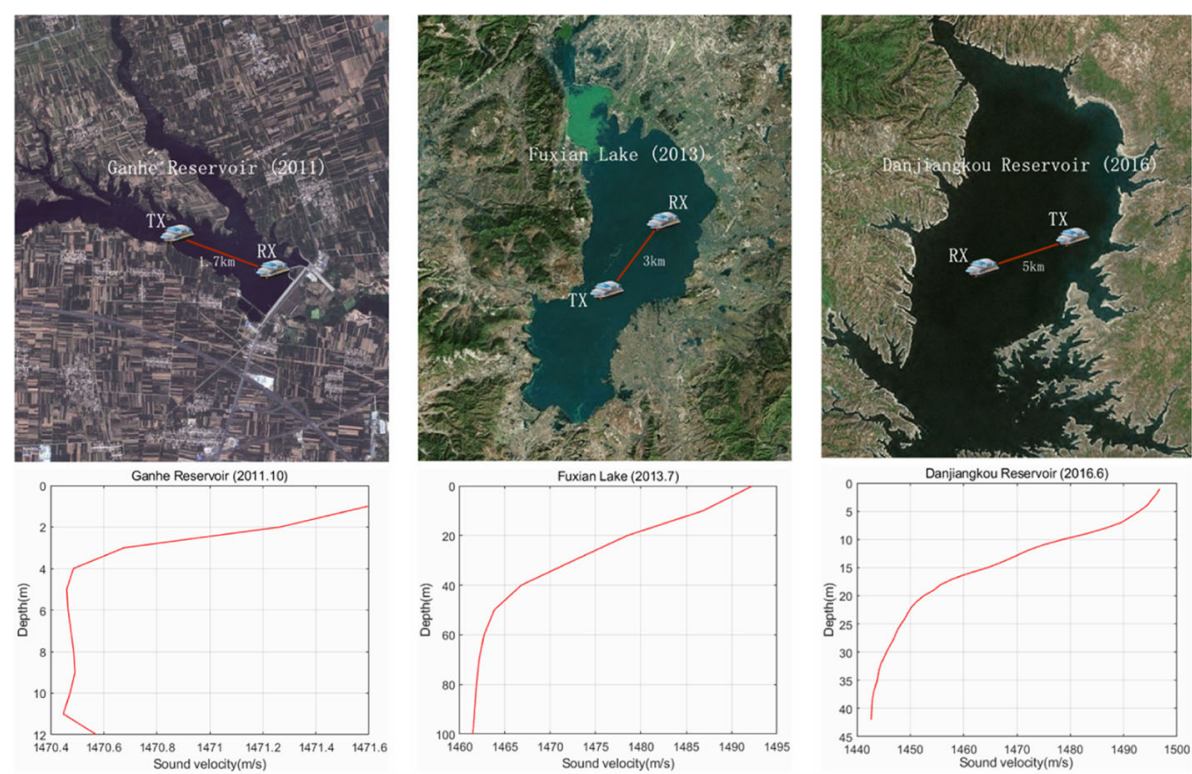

Fig. 9 Configuration of lake experiments 


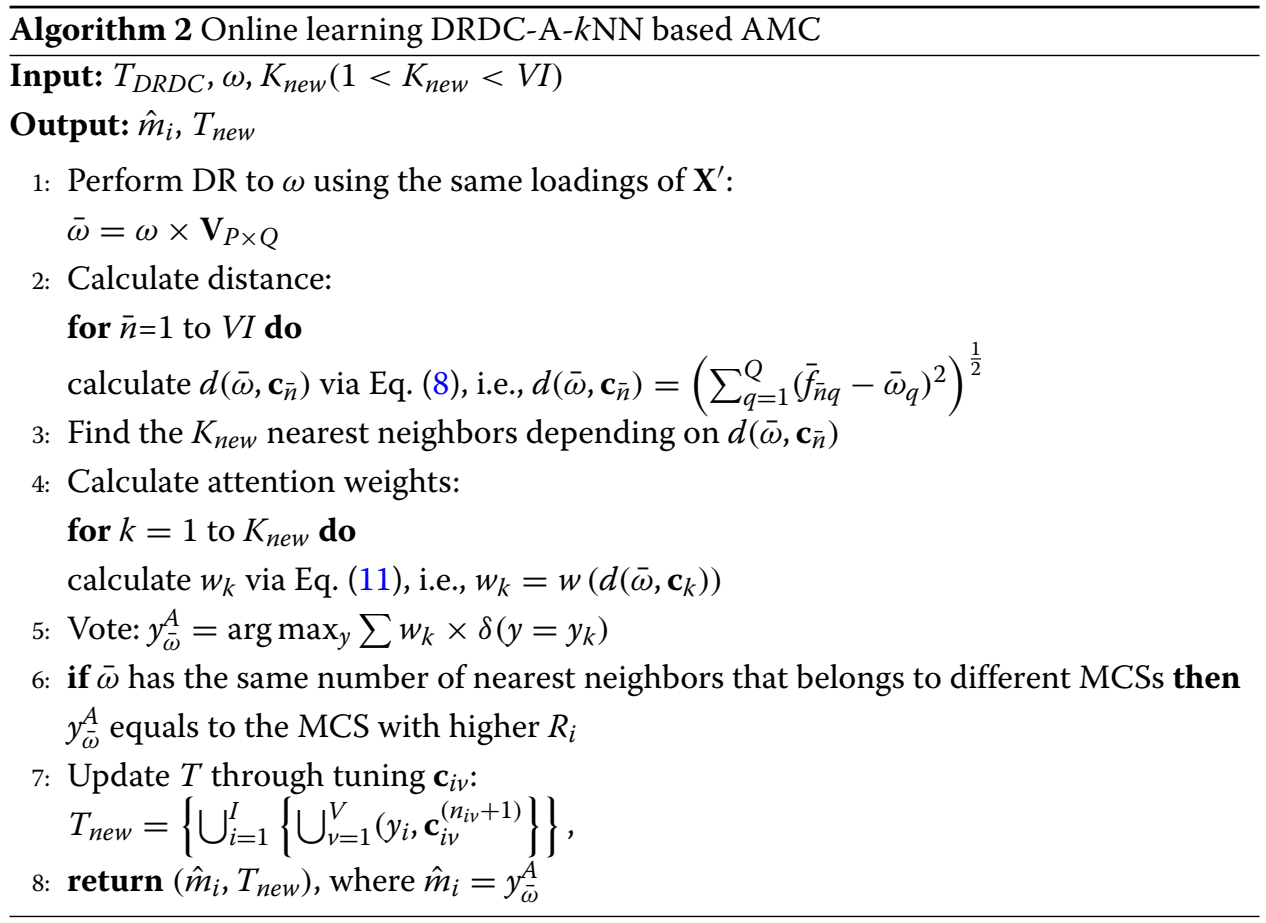

\section{Simulation results}

To evaluate the performance of the proposed two ML AMC approaches, several simulations have been conducted in CC-MC-MFSK UAC systems with three predefined MCSs, as depicted in Table 3. For data gathering, we collected a large set of real-world channel measurements from three previous field experiments conducted at Ganhe reservoir (October 2011), Fuxian lake (July 2013), and Danjiangkou reservoir (June 2016) [39]. Figure 9 shows the configurations, and Table 4 provides the mean value of each selected feature associated with these experiments. These data are then organized and labeled. Specifically, for each channel condition, the corresponding MCS is labeled by testing each MCS and selecting the best one according to Eq. (5). Eventually, a dataset of 1656 observations is made available, with labels covering all three MCS values.

Further, according to different simulation purposes, two categories of training sets are constructed as depicted in Tables 5 and 6, respectively. The first category is used to train and optimize the AMC classifier, aiming to validate the attention mechanism, select $k$ value, etc. To this end, each training set is a randomly extracted part from the whole 1656 observations. On the other hand, the second category is to evaluate the online learning ability of this AMC approach when deployed in practice, where each training set includes all the observations in a specific lake environment. Noticeably, throughout the simulations, we adopt the technique of $k$-fold cross-validation with $k=10$ [40], to calculate the corresponding classification accuracy $(\eta)$ for AMC.

Table 4 Lake environment

\begin{tabular}{lllllll}
\hline Index & Name & $\boldsymbol{\tau}_{\boldsymbol{m a x}}(\mathbf{m s})$ & $\boldsymbol{\tau}_{\boldsymbol{h m a x}}(\mathbf{m s})$ & $\boldsymbol{e}_{\mathbf{3}}$ & $\boldsymbol{e}_{\text {total }}$ & $\left|\boldsymbol{h}_{\mathbf{1}}\right|$ \\
\hline 1 & DJK-h12 & 6.9 & 6.6 & 0.24 & 2.25 & 0.27 \\
2 & FXH-h2 & 29.2 & 28.6 & 0.4 & 2.43 & 0.26 \\
3 & GH-h5 & 12.5 & 12 & 0.21 & 2.60 & 0.24 \\
\hline
\end{tabular}


Table 5 Category 1 of training sets

\begin{tabular}{lllllll}
\hline Index & $\mathbf{1}$ & $\mathbf{2}$ & $\mathbf{3}$ & $\mathbf{4}$ & $\mathbf{5}$ & $\mathbf{6}$ \\
\hline Sample number & 270 & 540 & 810 & 1080 & 1350 & 1656
\end{tabular}

\subsection{Analysis of the A-kNN AMC}

\subsubsection{Impact of different $k$ values}

As a key hyperparameter in $k \mathrm{NN}, k$ is the number of instances that are taken into account for the determination of affinity with different classes. However, a proper value of $k$ that leads to high prediction accuracy is challenging to be derived. Specifically, small $k$ values may increase undesired noise effects, while large values of $k$ will make the system computationally expensive or even produce errors when $k$ exceeds a certain value. In Fig. 10, given different $k$ values ranging from 1 to 55 , we investigate the performance of the A$k \mathrm{NN}$ classifier corresponding to all the training sets listed in Table 5 . Therein, $\eta$ is found to improve rapidly as $k$ increases at the beginning. However, this trend slows down and almost saturates when $k$ is greater than 15 . Consequently, we set $k$ to 15 in this work.

\subsubsection{A-kNN AMC versus traditional SNR-based AMC}

To better understand the nature of A- $k N N$ AMC performance, we learn the mapping function from the input channel conditions to the output MCSs by training an A- $k \mathrm{NN}$ classifier by training set 6 . The learned results are evaluated on test data in terms of the optimality of the predicted MCS (Fig. 11) and the achieved performances in terms of average throughput (aTP) and BER (Fig. 12), with comparison to a traditional modelbased method that only adopts SNR as the MCS switching metric. Therein, aTP at the $\varepsilon$ th SNR level is calculated via

$$
\operatorname{aTP}_{(\varepsilon)}=\frac{\eta}{N_{t(\varepsilon)}}\left(\sum_{i \in \hat{\mathcal{I}}} \hat{n}_{i(\varepsilon)}\left(1-\operatorname{mean}\left(B E R_{i(\varepsilon)}\right)\right) R_{i}\right)
$$

where $N_{t(\varepsilon)}$ denotes the total number of observations under such condition, while $\hat{\mathcal{I}}, \hat{n}_{i(\varepsilon)}$, and $B E R_{i(\varepsilon)}$ represent the set of indexes of the optimal MCSs, the number of correct optimal solutions in $m_{i}$, and the corresponding BER, respectively. Noticeably, since channels are represented by multidimensional features rather than a single SNR, each SNR may correspond to multiple optimal MCS choices with different data rates, and hence, aTP and BER do not vary monotonically in SNR. Instead, the BER curves stay rather flat around the required BER threshold, while the aTP improves as SNR increases. As confirmed by Figs. 11 and 12, the A-kNN AMC obtains near-ideal solutions in tracking channel dynamics under different operation scenarios, thanks to its immunity to channel modeling uncertainty and powerful multidimensional feature analysis capability. Therefore, our intelligent ML system is demonstrated to offer better AMC performance than its model-based counterpart, in terms of broad applicability to various operation scenarios.

Table 6 Category 2 of training sets

\begin{tabular}{llll}
\hline Index & $\mathbf{7}$ & $\mathbf{8}$ & $\mathbf{9}$ \\
\hline Lake environment & DJK-h12 & FXH-h2 & GH-h5 \\
Sample number & 468 & 1058 & 130 \\
\hline
\end{tabular}



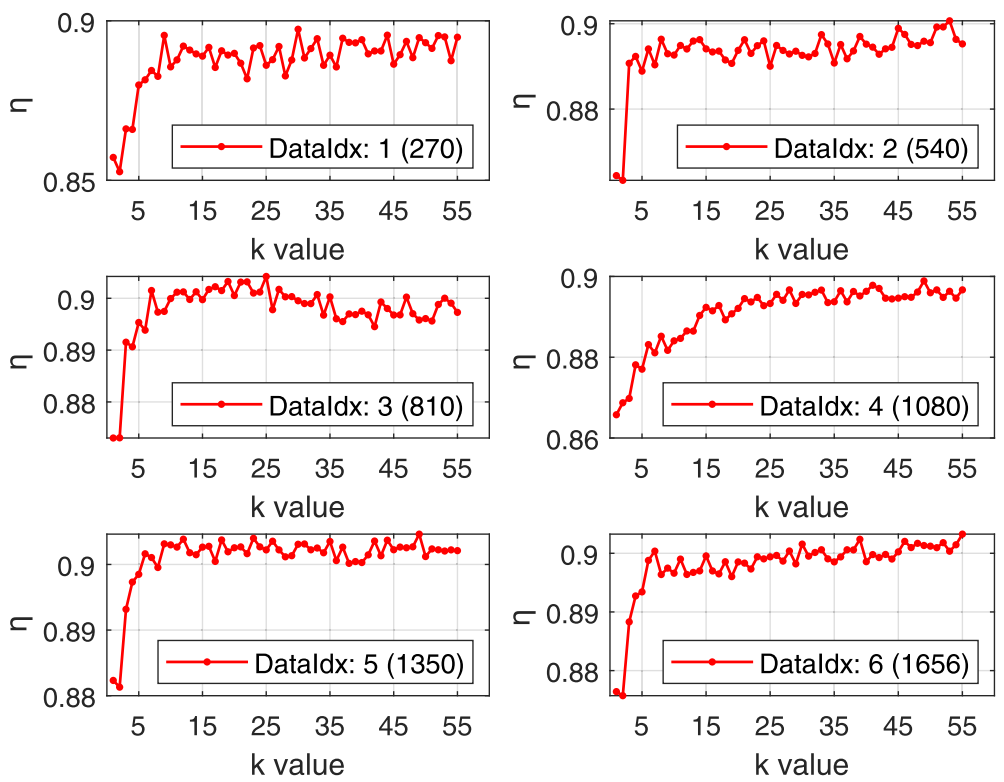

Fig. 10 Comparison of different $k$ values

\subsubsection{The learning curve of A-kNN AMC classifier}

Equipped with the online learning mechanism, the proposed AMC design has the capability of being adapted to various changing and unknown environments. To investigate whether it works in practice, we use the second category of data for further simulations. As illustrated in Fig. 13, an initial AMC classifier is built through offline training using training set 9 , which achieves a prediction accuracy of $90.4 \%$ in the UWA environment of GH. Next, we deploy this classifier to DJK-h12 (i.e., training set 7) and FXH-h2 (i.e., training set 8 ). Thanks to the learning ability, our AMC system is found to achieve a steadily improved prediction accuracy, and finally reaches an acceptable AMC performance, i.e., $\eta \geq 0.9$. Such results suggest the proposed online learning AMC classifier could extend its applicable scenarios intelligently.

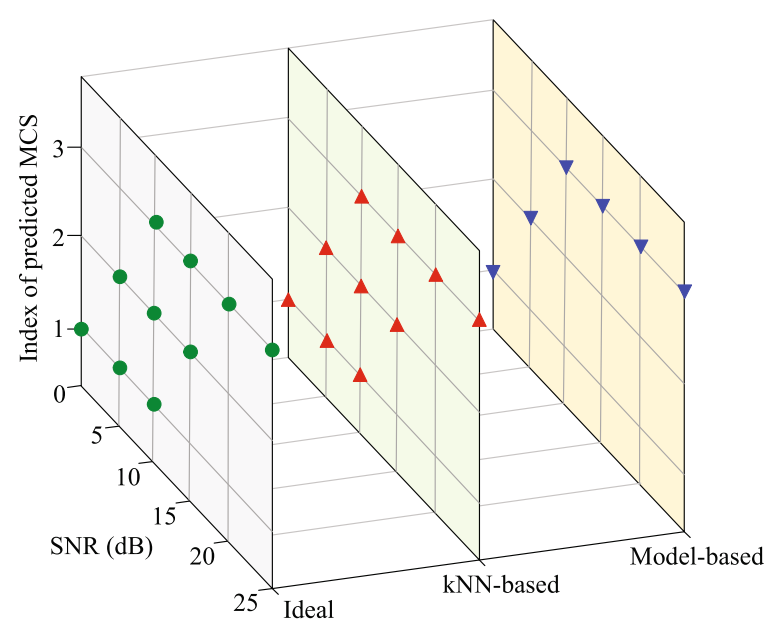

Fig. 11 Predicted optimal MCS vs SNR 

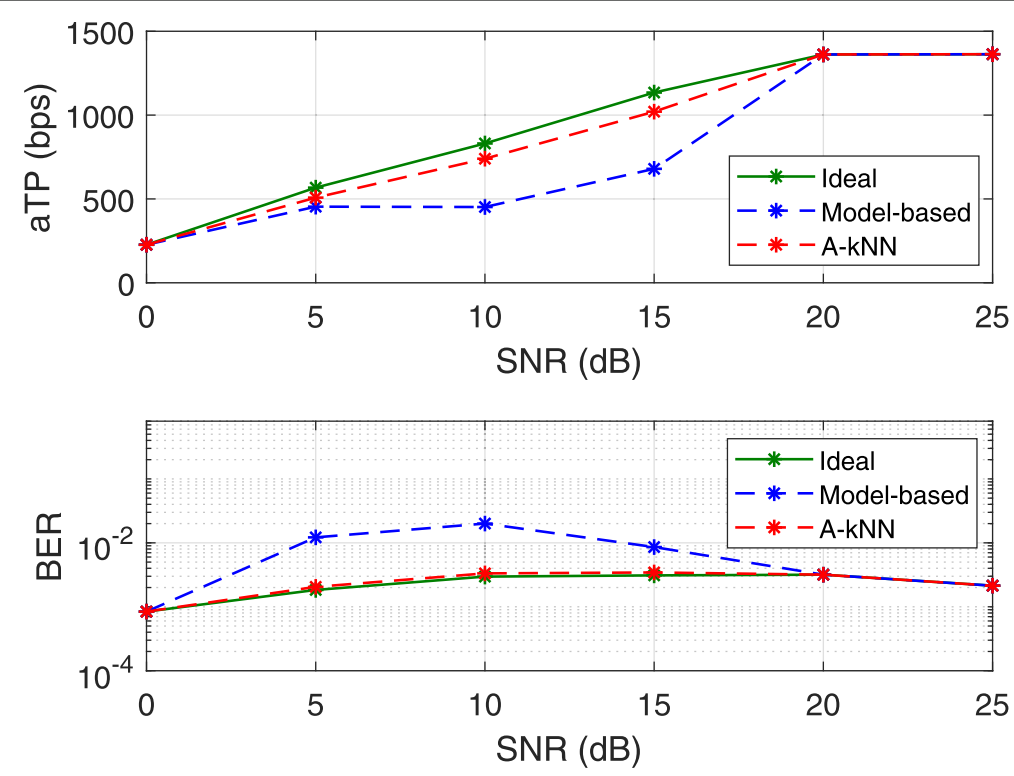

Fig. 12 aTP and BER vs SNR

\subsection{Analysis of the DRDC-A-kNN AMC}

\subsubsection{Effectiveness of DRDC}

First, the first category of training sets is adopted to evaluate the effectiveness of the DRDC processing. During the DR procedure, to determine the number of selected PCs, we adopt Eq. (20) and set $\psi=90 \%$, which indicates that the retained PCs cumulatively explain more than $90 \%$ of the total amount of information contained in the raw data. Therefore, according to the explained variance (EV) and cumulative explained variance (CEV) of PCs depicted in Fig. 14, the first three PCs are enough to satisfy Eq. (20). In addition, we present the PC loadings of training set 1 in Table 7, where each PC is a linear transformation of the original variables.

Once the dataset processed by DR is made available, we adopt the elbow method [41] to explore the optimal value of $V$ for the DC operation. Using training set 1 as an example, Fig. 15 shows $J_{c}$ as a function of different $V$, where $J_{c}$ denotes the cost function as

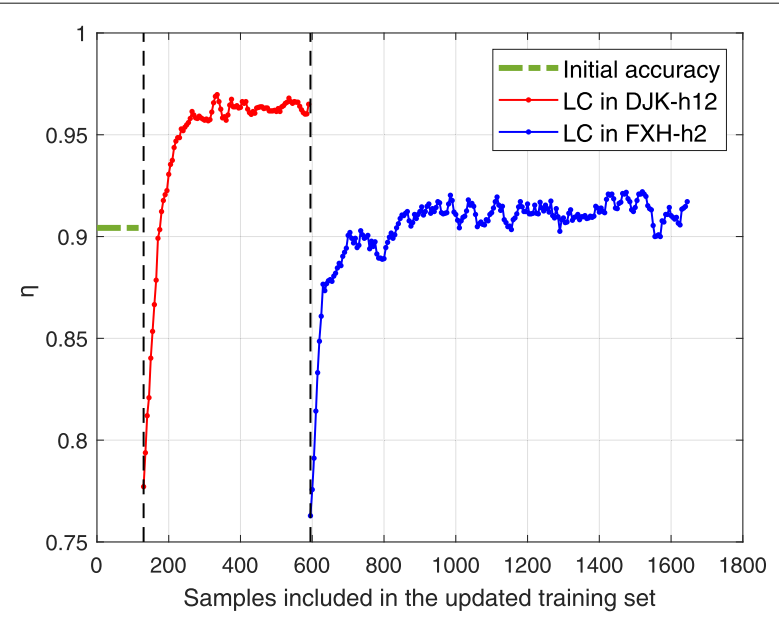

Fig. 13 The learning curve of A-kNN AMC classifier $(k=15)$ 


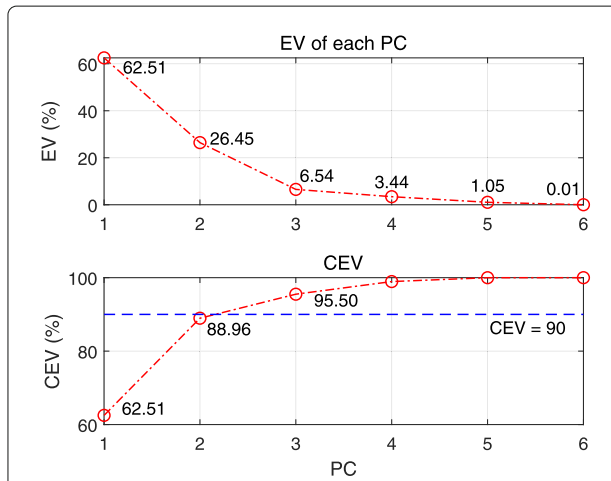

(a) Training set 1
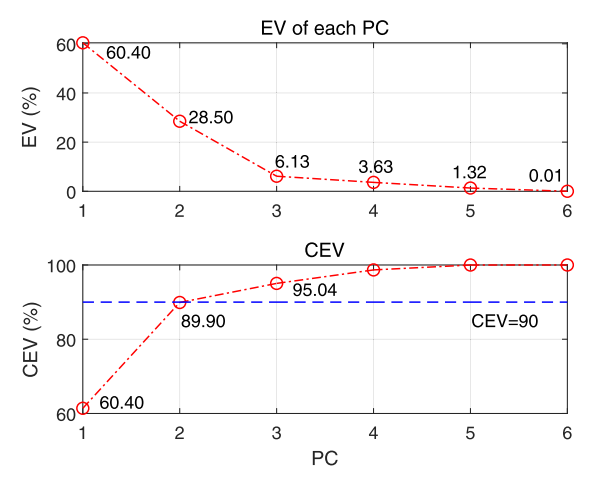

(c) Training set 3
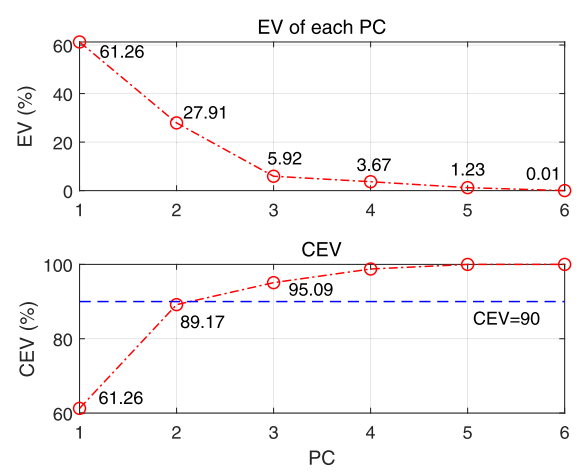

(e) Training set 5
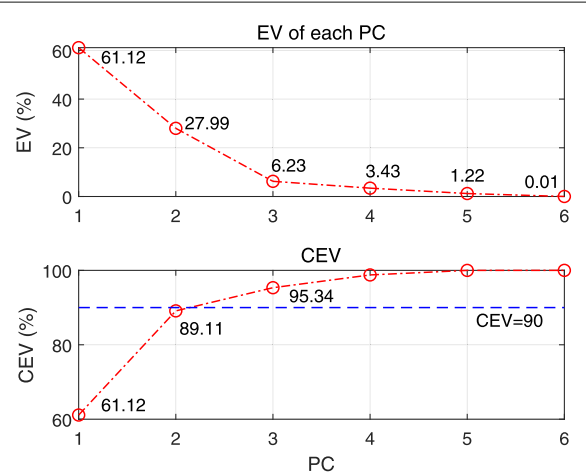

(b) Training set 2
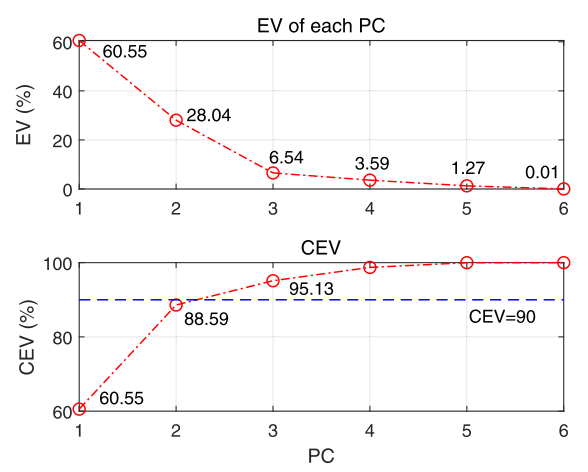

(d) Training set 4
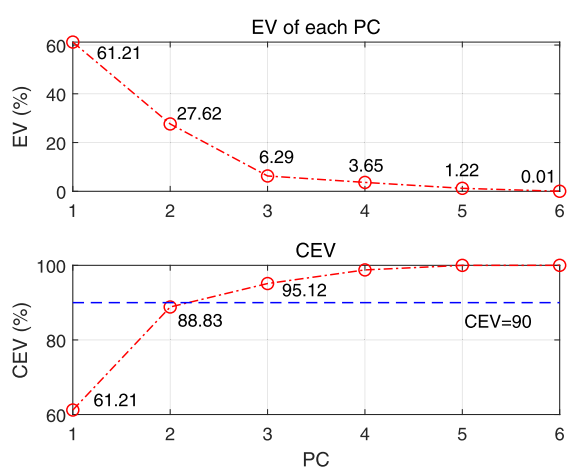

(f) Training set 6

Fig. 14 Explained variance of modified PCs by PCA (category 1 of training sets). a Training set 1. $\mathbf{b}$ Training set 2. c Training set 3. d Training set 4. e Training set 5. f Training set 6

Table 7 Loadings of retained PCs after DR (Trainingldx 1)

\begin{tabular}{lllllll}
\hline \multirow{2}{*}{ PCs } & \multicolumn{7}{c}{ Loadings } \\
\cline { 2 - 7 } & SNR & $\boldsymbol{\tau}_{\max }$ & $\boldsymbol{\tau}_{\text {hmax }}$ & $\boldsymbol{e}_{\mathbf{3}}$ & $\boldsymbol{e}_{\text {total }}$ & $\mid \boldsymbol{h}_{\mathbf{1}} \boldsymbol{|}$ \\
\hline 1 & -0.0165 & 0.6872 & 0.6894 & -0.1260 & 0.1733 & -0.0796 \\
2 & 0.9984 & 0.0186 & 0.0192 & 0.0438 & -0.0252 & -0.0040 \\
3 & -0.0360 & 0.0958 & 0.0831 & 0.8604 & 0.1296 & 0.4749 \\
\hline
\end{tabular}




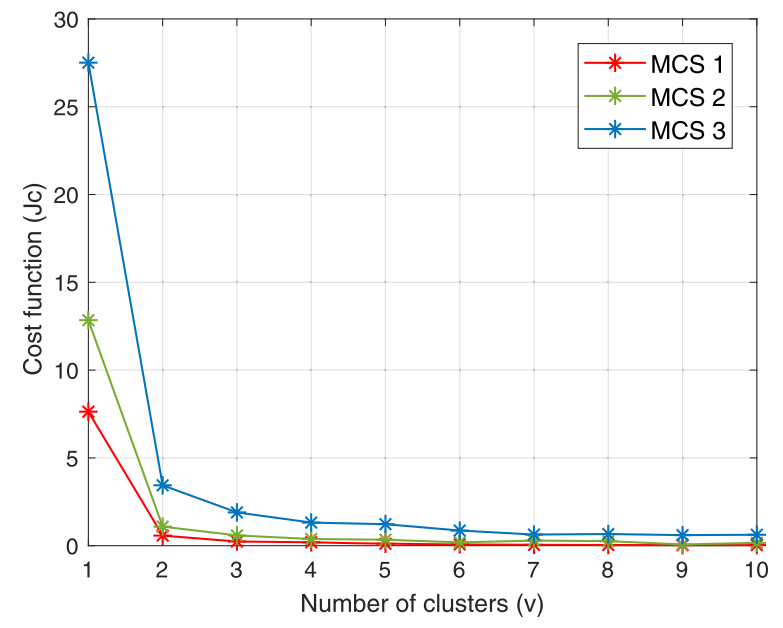

Fig. 15 Determine the value of $V$ (training set 1)

$$
J_{c}=\sum_{v=1}^{V} \sum_{\mathbf{o}_{i v} \in S_{i v}}\left\|\mathbf{o}_{i v}-\mathbf{c}_{i v}\right\|_{2}^{2},
$$

which is the sum of squared errors (SSE) of samples in each cluster corresponding to the centroid $\mathbf{c}_{i v}$. Remarkably, it can be seen that, with the increase of $V$, the curves first drop sharply and then slowly approach zero. Aiming at finding a good trade-off between $J_{c}$ and $V$, we set the optimal $V$ for each $m_{i}$ to 2, which is the elbow of the curves and represents that our returns will diminish as $V$ continuously increases [41]. Further, by the same analysis, $V$ for the other training sets also holds the same optimal value.

Table 8 compares the training performance with and without DRDC, in terms of prediction accuracy and system complexity. As expected, through performing the DRDC processing, our classifier achieves a significant reduction in complexity by nearly $190.8 \%$ at the price of an average accuracy loss of $2.5 \%$. Moreover, given the online learning during the actual deployment, DRDC will play an even more crucial role in system efficiency improvement as the training set keeps expanding.

Table 8 Performance comparison between training with and without DRDC ( $\left.k_{\text {new }}=3, V=2\right)$

\begin{tabular}{|c|c|c|c|c|c|}
\hline Dataldx & DRDC & Accuracy (\%) & $\begin{array}{l}\text { Accuracy } \\
\text { loss }\end{array}$ & $\begin{array}{l}\text { Prediction } \\
\text { speed (obs/sec) }\end{array}$ & $\begin{array}{l}\text { Reduced } \\
\text { complexity }\end{array}$ \\
\hline \multirow{2}{*}{1} & Off & 89.2 & \multirow{2}{*}{$4.5 \%$} & 5077 & \multirow{2}{*}{$67.7 \%$} \\
\hline & On & 84.7 & & 8512 & \\
\hline \multirow{2}{*}{2} & Off & 89.3 & \multirow{2}{*}{$0.9 \%$} & 3779 & \multirow{2}{*}{$120.6 \%$} \\
\hline & On & 88.4 & & 8337 & \\
\hline \multirow{2}{*}{3} & Off & 90.1 & \multirow{2}{*}{$3.6 \%$} & 2912 & \multirow{2}{*}{$189.3 \%$} \\
\hline & On & 86.5 & & 8424 & \\
\hline \multirow{2}{*}{4} & Off & 89.0 & \multirow{2}{*}{$2.5 \%$} & 2657 & \multirow{2}{*}{$215.9 \%$} \\
\hline & On & 86.5 & & 8393 & \\
\hline \multirow{2}{*}{5} & Off & 90.1 & \multirow{2}{*}{$1.6 \%$} & 2358 & \multirow{2}{*}{$259.1 \%$} \\
\hline & On & 88.5 & & 8467 & \\
\hline \multirow{3}{*}{6} & Off & 89.6 & \multirow{2}{*}{$1.8 \%$} & 2169 & \multirow{2}{*}{$292.3 \%$} \\
\hline & On & 87.8 & & 8509 & \\
\hline & $\mathrm{Me}$ & & $2.5 \%$ & & $190.8 \%$ \\
\hline
\end{tabular}




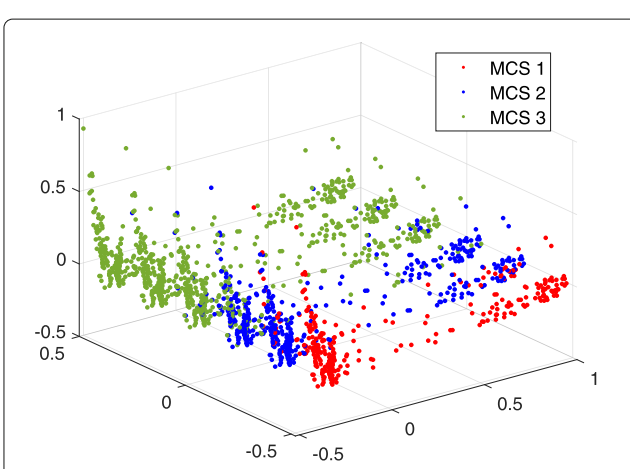

(a) Training set after DR

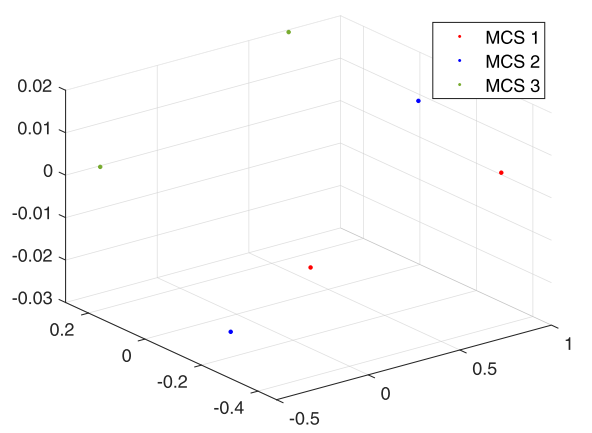

(b) Training set after DR+DC

Fig. 16 Variation of training set along with DRDC (training set 6). a Training set after DR. b Training set after $\mathrm{DR}+\mathrm{DC}$

To make the DRDC process more intuitive, Fig. 16 shows the detailed variation of training set 6 along with such processing, where the observations of different MCSs are represented in different colors. The DR operation first converts the sophisticated sixdimensional original samples to a visualized new set of only three dimensionalities. Then further processed by DC, the previous 1656 six-dimensional observations are successfully represented by only six data points with three dimensionalities, thus offering an excellent efficiency enhancement to our ML-aided AMC system.

\subsubsection{Learning curve of the DRDC-A-kNN AMC classifier}

Following the same simulation procedure, Fig. 17 presents the learning curve of the DRDC-A- $k N N$ AMC classifier and compares the performance with that of its A- $k N N$ counterpart. Remarkably, despite the introduction of DRDC, the prediction accuracy of our ML-aided AMC classifier suffers only a slight loss, which is no more than $10 \%$. Moreover, the DRDC-A- $k$ NN AMC classifier is much more efficient and can still maintain excellent learning ability, thus enabling a continuously increased applicability in the actual deployment.

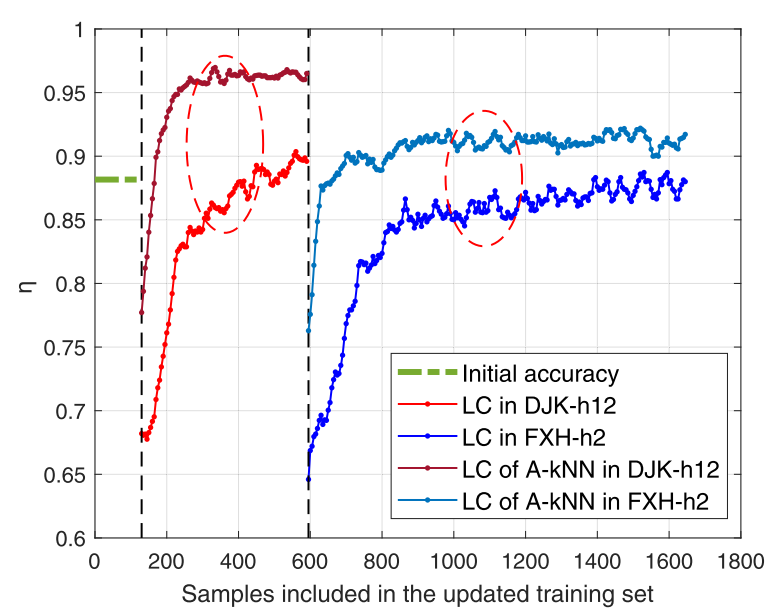

Fig. 17 Learning curve of the DRDC-A-kNN AMC classifier $\left(k_{\text {new }}=3, V=2\right)$ 


\title{
6 Conclusion and future work
}

This article turns to an ML perspective to cope with the major challenges of AMC design in the harsh underwater environment. The proposed online learning A- $k \mathrm{NN}$ classifier based on SL enables a novel implementation of AMC, which has excellent immunity to channel modeling uncertainty. Moreover, to handle the inherent high-complexity issues, we further present the DRDC-A- $k N N$ classifier for feature dimensionality reduction and data condensation, which can offer a great complexity reduction compared to the A- $k \mathrm{NN}$ approach, and facilitate an easier implementation of the AMC systems.

While the proposed two ML methods expand the applicability of AMC systems in UACs compared with the traditional model-based approaches, there are still ample issues left for future work. Currently, in order to reduce the demand for computing resources and training time, the features used to train the AMC model are manually extracted from CSI by experience. However, the uncertainty of experience may potentially impact on the system performance, since there are no best-practice rules on which features are crucial for MCS switching in underwater AMC. To alleviate this problem, it may be necessary to investigate a DL-based AMC framework that can be categorized as a totally data-driven solution, to enable sustainable model improvement through automatically detecting and generating more complex and high-level features from raw data sources.

\footnotetext{
Abbreviations

UAC: Underwater acoustic communication; UWA: Underwater acoustic; ML: Machine learning; AMC: Adaptive modulation and coding; A-kNN: Attention-aided k-nearest neighbor; DRDC-A-kNN: Dimensionality-reduced and data-clustered A-kNN; PHY: Physical layer; MCS: Modulation and coding scheme; CSI: Channel state information; DL: Deep learning; DNN: Deep neural network; TX: Transmitter; RX: Receiver; SSP: Sound speed profile; SLI: Statistical link information; SL: Supervised learning; UL: Unsupervised learning; SSL: Semi-supervised learning; RL: Reinforcement learning.
}

Acknowledgements

The authors would like to thank all the referees for their constructive and insightful comments on this paper.

\begin{abstract}
Authors' contributions
Conceptualization, L.H. and Z.T.; methodology, L.H. and Z.T.; software, L.H.; validation, Q.Z.; formal analysis, L.H.; investigation, L.H. and L.Z.; data curation, L.H.; writing—original draft preparation, L.H.; writing-review and editing, W.T., Y.W., and C.H.; visualization, L.H. All authors read and approved the final manuscript.

\section{Funding}

This work was supported in part by the Science, Technology and Innovation Commission of Shenzhen Municipality (Grant No. JCYJ20180306170932431), in part by the National Key R\&D Program of China (Grant No. 2016YFC1400203), in part by the National Natural Science Foundation of China (Grant Nos. 61771394, 61531015, and 61801394), and in part by the Natural Science Basic Research Plan in Shaanxi Province of China (Grant No. 2018JM6042).
\end{abstract}

\section{Availability of data and materials}

The datasets used and/or analyzed during the current study are available from the corresponding author on reasonable request.

\section{Competing interests}

The authors declare that they have no competing interests.

\section{Author details}

${ }^{1}$ Research and Development Institute, Northwestern Polytechnical University in Shenzhen, Shenzhen, 518057, China.

${ }^{2}$ School of Marine Science and Technology, Northwestern Polytechnical University, Xi'an, 710072, Shaanxi, China. ${ }^{3}$ State Key Laboratory of Public Big Data, Guizhou University, Guiyang, 550025, Guizhou, China. ${ }^{4}$ Department of Electrical and Computer Engineering, George Mason University, Fairfax, VA, 22030, USA.

Received: 5 May 2020 Accepted: 29 September 2020

Published online: 17 October 2020

\section{References}

1. I. F. Akyildiz, D. Pompili, T. Melodia, Underwater acoustic sensor networks: research challenges. Ad Hoc Netw. 3(3), 257-279 (2005)

2. M. Badiey, Y. Mu, J. A. Simmen, S. E. Forsythe, Signal variability in shallow-water sound channels. IEEE J. Oceanic Eng. 25(4), 492-500 (2000) 
3. M. Stojanovic, J. Preisig, Underwater acoustic communication channels: propagation models and statistical characterization. IEEE Commun. Mag. 47(1), 84-89 (2009)

4. A. Song, M. Badiey, H. Song, W. S. Hodgkiss, M. B. Porter, the KauaiEx Group Impact of ocean variability on coherent underwater acoustic communications during the Kauai experiment (KauaiEx). J. Acoust. Soc. Am. 123(2), 856-865 (2008)

5. M. Chitre, S. Shahabudeen, L. Freitag, M. Stojanovic, in OCEANS 2008, Recent advances in underwater acoustic communications \& networking (IEEE, Quebec City, 2008), pp. 1-10

6. J. Hayes, Adaptive feedback communications. IEEE Trans. Commun. Technol. 16(1), 29-34 (1968)

7. W. T. Webb, QAM: the modulation scheme for future mobile radio communications?. Electron. \& Commun. Eng. J. 4(4), 167-176 (1992)

8. W. Webb, R. Steele, Variable rate QAM for mobile radio. IEEE Trans. Commun. 43(7), 2223-2230 (1995)

9. M. Rajesh, B. Shrisha, N. Rao, H. Kumaraswamy, in 2016 IEEE Int'l Conf. Recent Trends in Electron., Inform. \& Commun. Technol. (RTEICT), An analysis of BER comparison of various digital modulation schemes used for adaptive modulation (IEEE, Bangalore, 2016), pp. 241-245

10. A. Svensson, An introduction to adaptive QAM modulation schemes for known and predicted channels. P. IEEE. 95(12), 2322-2336 (2007)

11. Y. Yang, H. Ma, S. Aissa, Cross-layer combining of adaptive modulation and truncated ARQ under cognitive radio resource requirements. IEEE Trans. Veh. Technol. 61(9), 4020-4030 (2012)

12. K.-H. Park, M. A. Imran, P. Casari, H. Kulhandjian, H. Chen, A. Abdi, F. Dalgleish, IEEE Access special section editorial: underwater wireless communications and networking. IEEE Access. 7, 52288-52294 (2019)

13. A. Benson, J. Proakis, M. Stojanovic, in OCEANS 2000 MTS/IEEE Conf. and Exhibition. Conf. Proc. (Cat. No. 00CH37158), Towards robust adaptive acoustic communications, vol. 2 (IEEE, Providence, 2000), pp. 1243-1249

14. L. Wan, H. Zhou, X. Xu, Y. Huang, S. Zhou, Z. Shi, J.-H. Cui, Adaptive modulation and coding for underwater acoustic OFDM. IEEE J. Oceanic Eng. 40(2), 327-336 (2015)

15. X. Shen, J. Huang, Q. Zhang, C. He, Achieving high speed UWA communication with adaptive MOMC technology. J. Northwestern polytechnical university. 25(1), 147 (2007)

16. M. J. Er, Y. Zhou, Theory and Novel Applications of Machine Learning. (IntechOpen, 2009)

17. H. Ye, G. Y. Li, B.-H. Juang, IEEE Wirel. Commun. Le. 7(1), 114-117 (2018)

18. C. Luo, J. Ji, Q. Wang, X. Chen, P. Li, Channel state information prediction for $5 \mathrm{G}$ wireless communications: a deep learning approach. IEEE Trans. Netw. Sci. Eng. 7(1), 227-236 (2018)

19. Y. Wang, J. Yang, M. Liu, G. Gui, Lightamc: lightweight automatic modulation classification using deep learning and compressive sensing. IEEE Trans. Veh. Technol. 69(3), 3491-3495 (2020)

20. T. Hu, Y. Fei, in 2010 IEEE Int'l Symp. Model., Anal. and Simul. of Comput. and Telecommun. Syst, An adaptive and energy-efficient routing protocol based on machine learning for underwater delay tolerant networks (IEEE, Miami Beach, 2010), pp. 381-384

21. K. Pelekanakis, L. Cazzanti, G. Zappa, J. Alves, in 2016 IEEE Third Underwater Commun. and Netw. Conf. (UComms), Decision tree-based adaptive modulation for underwater acoustic communications (IEEE, Lerici, 2016), pp. 1-5

22. C. Wang, Z. Wang, W. Sun, D. R. Fuhrmann, Reinforcement learning-based adaptive transmission in time-varying underwater acoustic channels. IEEE Access. 6, 2541-2558 (2018)

23. J. Heidemann, M. Stojanovic, M. Zorzi, Underwater sensor networks: applications, advances and challenges. Philos. T. R. Soc. A. 370(1958), 158-175 (2012)

24. L. R. LeBlanc, F. H. Middleton, An underwater acoustic sound velocity data model. J. Acoust. Soc. Am. 67(6), 2055-2062 (1980)

25. A. Ahmed, M. Younis, in 2017 IEEE Int'I Conf. Commun. (ICC), Distributed real-time sound speed profiling in underwater environments, (Paris, 2017), pp. 1-7

26. A. J. Goldsmith, S.-G. Chua, Adaptive coded modulation for fading channels. IEEE Trans. Commun. 46(5), 595-602 (1998)

27. L. Huang, L. Zhang, Y. Wang, Q. Zhang, in 2019 IEEE International Conference on Signal, Information and Data Processing (ICSIDP), Chongqing, China, A Two-dimensional Strategy of Adaptive Modulation and Coding for Underwater Acoustic Communication Systems, (2019), pp. 1-5

28. A. Misra, V. Krishnamurthy, S. Schober, in IEEE 6th Workshop on Signal Process. Adv. in Wirel. Commun., 2005., Stochastic learning algorithms for adaptive modulation (IEEE, New York, 2005), pp. 756-760

29. C. M. Biship, Pattern recognition and machine learning (information science and statistics). (Springer-Verlag New York, Inc., New York, 2006)

30. G. W. Lindsay, Attention in psychology, neuroscience, and machine learning. Front. Comput. Neurosc. 14, 29 (2020)

31. M. Luong, H. Pham, C. D. Manning, in P. 2015 Conf. Empir. Methods in Nat. Lang. Process. (emnlp), Effective approaches to attention-based neural machine translation (ACL, Lisbon, 2015), pp. 1412-1421

32. J. Han, L. Zhang, Q. Zhang, G. Leus, Low-complexity equalization of orthogonal signal-division multiplexing in doubly-selective channels. IEEE Trans. Signal Proces. 67(4), 915-929 (2018)

33. J. Han, Y. Wang, L. Zhang, G. Leus, Time-domain oversampled orthogonal signal-division multiplexing underwater acoustic communications. J. Acoust. Soc. Am. 145(1), 292-300 (2019)

34. J. Han, S. P. Chepuri, Q. Zhang, G. Leus, Iterative per-vector equalization for orthogonal signal-division multiplexing over time-varying underwater acoustic channels. IEEE J. Oceanic Eng. 44(1), 240-255 (2019)

35. R. Sinha, R. D. Yates, in Veh. Technol. Conf. Fall 2000. IEEE VTS Fall VTC2000. 52nd Veh. Technol. Conf. (Cat. No. 00CH37152), An OFDM based multicarrier MFSK system, vol. 1 (IEEE, Boston, 2000), pp. 257-264

36. C. X. Gao, H. X. Yang, F. Yuan, E. Cheng, Underwater acoustic communication system based on MC-MFSK. Appl. Mech. and Mater. 556, 4897-4900 (2014). Trans Tech Publ

37. K. Beyer, J. Goldstein, R. Ramakrishnan, U. Shaft, in Int'l Conf. Database Theory, When is "nearest neighbor" meaningful? (Springer, Berlin, 1999), pp. 217-235

38. H. Zou, T. Hastie, R. Tibshirani, Sparse principal component analysis. J. Comput. Graph. Stat. 15(2), $265-286$ (2006) 
39. T. Yang, S. Huang, in P. the 11th ACM Int'l Conf. Underwater Netw. \& Syst, Building a database of ocean channel impulse responses for underwater acoustic communication performance evaluation: issues, requirements, methods and results (ACM, Shanghai, 2016), pp. 1-8

40. T. T. Wong, P. Y. Yeh, Reliable accuracy estimates from k-fold cross validation. IEEE Trans. Knowl. Data En. 32(8), 1586-1594 (2020)

41. T. M. Kodinariya, P. R. Makwana, Review on determining number of cluster in k-means clustering. Int. J. Adv. Res. Comput. Sci. Manage. Stud. 1(6), 90-95 (2013)

\section{Publisher's Note}

Springer Nature remains neutral with regard to jurisdictional claims in published maps and institutional affiliations.

Submit your manuscript to a SpringerOpen ${ }^{\odot}$ journal and benefit from:

- Convenient online submission

Rigorous peer review

Open access: articles freely available online

- High visibility within the field

- Retaining the copyright to your article

Submit your next manuscript at $\boldsymbol{\triangleright}$ springeropen.com 